OPEN ACCESS

Edited by:

Fabio Bagnoli,

GlaxoSmithKline, Italy

Reviewed by:

Arun Kumar,

Coalition for Epidemic Preparedness Innovations (CEPI), Norway

Randy A. Albrecht,

Icahn School of Medicine at Mount

Sinai, United States

*Correspondence:

Cynthia Calzas

cynthia.calzas@inra.fr

Christophe Chevalier

christophe.chevalier@inra.fr

Specialty section:

This article was submitted to Vaccines and Molecular Therapeutics,

a section of the journal

Frontiers in Immunology

Received: 15 January 2019 Accepted: 27 June 2019

Published: 17 July 2019

Citation:

Calzas C and Chevalier C (2019)

Innovative Mucosal Vaccine Formulations Against Influenza A Virus Infections. Front. Immunol. 10:1605. doi: 10.3389/fimmu.2019.01605

\section{Innovative Mucosal Vaccine Formulations Against Influenza A Virus Infections}

\author{
Cynthia Calzas* and Christophe Chevalier* \\ VIM, UR892, Equipe Virus Influenza, INRA, University PARIS-SACLAY, Jouy-en-Josas, France
}

Despite efforts made to develop efficient preventive strategies, infections with influenza A viruses (IAV) continue to cause serious clinical and economic problems. Current licensed human vaccines are mainly inactivated whole virus particles or split-virion administered via the parenteral route. These vaccines provide incomplete protection against IAV in high-risk groups and are poorly/not effective against the constant antigenic drift/shift occurring in circulating strains. Advances in mucosal vaccinology and in the understanding of the protective anti-influenza immune mechanisms suggest that intranasal immunization is a promising strategy to fight against IAV. To date, human mucosal anti-influenza vaccines consist of live attenuated strains administered intranasally, which elicit higher local humoral and cellular immune responses than conventional parenteral vaccines. However, because of inconsistent protective efficacy and safety concerns regarding the use of live viral strains, new vaccine candidates are urgently needed. To prime and induce potent and long-lived protective immune responses, mucosal vaccine formulations need to ensure the immunoavailability and the immunostimulating capacity of the vaccine antigen(s) at the mucosal surfaces, while being minimally reactogenic/toxic. The purpose of this review is to compile innovative delivery/adjuvant systems tested for intranasal administration of inactivated influenza vaccines, including micro/nanosized particulate carriers such as lipid-based particles, virus-like particles and polymers associated or not with immunopotentiatory molecules including microorganism-derived toxins, Toll-like receptor ligands and cytokines. The capacity of these vaccines to trigger specific mucosal and systemic humoral and cellular responses against IAV and their (cross)-protective potential are considered.

\section{Keywords: influenza A virus, mucosal vaccines, adjuvant, delivery systems, intranasal immunization}

\section{INTRODUCTION}

Despite progress in antiviral therapies, influenza viruses remain an important cause of respiratory tract (RT) infections in humans and animals worldwide (1). Influenza viruses are members of the Orthomyxoviridae family and are classified into four genera (A, B, C, D). Influenza A viruses (IAV), whose natural reservoirs are aquatic birds, can infect a broad spectrum of animal species including humans and poultry. Based on the molecular structure and genetic characteristics of the surface glycoproteins hemagglutinin (HA) and neuraminidase (NA), IAV can be categorized into $18 \mathrm{HA}$ subtypes and 11 NA subtypes. 
IAV have a negative-sense, single-stranded RNA genome consisting of 8 segments encoding for at least 17 viral proteins (1). Each segment is associated with the viral nucleoprotein (NP) and the three polymerase components, namely the polymerase basic protein 1 (PB1) and 2 (PB2) and the polymerase acidic protein (PA). These ribonucleoprotein complexes are encapsidated by the matrix protein 1 (M1) beneath an envelope composed of a lipid bilayer derived from the host plasma membrane where are embedded the surface glycoproteins HA, NA and the matrix protein 2 (M2). HA is responsible for the binding of the virus to sialic acid moieties at the host cell surface. HA is a trimeric glycoprotein and each monomer is composed of two domains, a globular head (HA1) and a stalk domain (HA2). HA1, exposed at the surface of the virion is subject to a high degree of antigenic variations. HA2, more conserved across IAV, is involved in various steps of the virus life cycle, including the fusion between the viral envelope and the endosomal host membrane. NA is a tetrameric glycoprotein which enzymatically removes sialic acid residues from the surface of infected cells, allowing the release of budding virions. $\mathrm{M} 2$ is a tetrameric protein acting as a protonselective ion channel which triggers the uncoating of the viral

Abbreviations: Ab, antibody; Ag, antigen; APC, antigen-presenting cell; ASC, antibody-secreting cell; BALT, bronchus-associated lymphoid tissues; BCR, B cell receptor; CCL, (C-C motif) ligand; CCR, (C-C motif) receptor; CCS, ceramide carbamoyl-spermine; cDC, conventional dendritic cell; c-diAMP, cyclic-di-adenosine monophosphate; cGAMP, 2,3'-cyclic-guanosine monophosphate-adenosine monophosphate; CDN, cyclic di-nucleotides; CpG-ODN, synthetic oligodeoxynucleotides composed of unmethylated $\mathrm{CPG}$ motifs; CT, cholera toxin; CTA1, A1 subunit of the cholera toxin; CTA1$\mathrm{DD}, \mathrm{CTA} 1$ fused to a synthetic dimer of the Ig binding D domain (DD) from Staphylococcus aureus protein A; CTL, cytotoxic T lymphocyte; DC, dendritic cell; DDAB, dimethyldioctadecylammonium bromide; DLN, draining lymph nodes; DMPC, dimyristoyl phosphatidylcholine; DMPG, dimyristoyl phosphatidylglycerol; DOTAP, dioleoyl-3-trimethylammoniumpropane; DPPC, dipalmitoyl phosphatidylcholine; FAE, follicle-associated epithelium; GC, germinal center; HA, hemagglutinin; HA1, globular head domain of hemagglutinin; HA2, stalk domain of hemagglutinin; HAI, hemagglutinationinhibition; HIV, human immunodeficiency virus; HPAIV, highly pathogenic avian influenza viruses; HTCC, $N$-[(2-hydroxy-3-trimethylammonium) propyl] chitosan chloride; IAV, influenza A viruses; IBV, influenza B virus; IFN, interferon; Ig, immunoglobulins; IIV, inactivated influenza viruses; IL, interleukin; i.n., intranasal; ISCOM, immune stimulating complex; ISCOMATRIX, immune stimulating complex matrix; LAIV, live-attenuated influenza viruses; LPAIV, low pathogenic avian influenza viruses; LRT, lower respiratory tract; LT, Escherichia coli heat-labile toxin; M cells, microfold cells; M1, matrix protein 1; M2(e), (ectodomain of the) matrix protein 2; MALT, mucosa-associated lymphoid tissues; mDC, myeloid dendritic cell; MHC, major histocompatibility complex; moDC, monocyte-derived dendritic cell; MPLA, monophosphoryl lipid A; NA, neuraminidase; NAIP, nucleotide oligomerization domain-like receptor family, apoptosis inhibitory protein; NALT, nasopharynx-associated lymphoid tissues; NK, natural killer; NLR, nucleotide oligomerization domain-like receptor; NLRP3, nucleotide oligomerization domain-like receptor family, pyrin domain containing 3; NP, nucleoprotein; $\mathrm{N}_{\mathrm{RSV}}$, nucleoprotein of the respiratory syncytial virus; PA, polymerase acidic protein; PAMP, pathogen associated molecular pattern; PB1, polymerase basic protein 1; $\mathrm{PB} 2$, polymerase basic protein 2; $\mathrm{pDC}$, plasmacytoid dendritic cell; PEI, polyethyleneimine; PG, phosphatidylglycerol; $\gamma$-PGA, poly ( $\gamma$-glutamic acid); PLGA, poly (D, L-lactide-co-glycolide); poly (I:C), polyinosinepolycytidylic acid; PRR, pattern recognition receptor; RT, respiratory tract; SP-C, surfactant protein C; STING, stimulator of interferon genes; Th, T helper; TLR, Toll-like receptor; TMC, $\mathrm{N}$-trimethyl-derivatives of chitosan; TRIF, Toll or interleukin-1 receptor domain-containing adaptor-inducing interferon- $\beta ; T_{R M}$, resident memory T cell; URT, upper respiratory tract; VLP, virus-like particle; WIV, whole inactivated viruses. ribonucleoprotein complexes necessary for the release of the viral genetic material into the host cytosol. Unlike HA and NA, the ectodomain of M2 (M2e) is sparsely expressed at the surface of the virion, less subjected to the host immune pressure and consequently more conserved across IAV (1).

Circulating IAV are continuously evolving, leading to the emergence of new strains expressing surface glycoproteins that have distinct antigenic properties (1). In particular, point mutations in the viral genome RNA result in the emergence of new strains responsible for seasonal epidemics ("antigenic drift"), and the co-infection of a host with multiple IAV strains can result in genetic reassortments responsible for the emergence of novel subtypes ("antigenic shift") that can give rise to strains with pandemic potential. The disease severity caused by IAV infections depends on several parameters such as viral and host factors. In humans, the virus initially targets the mucosa of the upper RT (URT) (nose, pharynx), leading to dry cough, nasal discharge, rhinitis, pharyngitis and fever, and can eventually reach the lower RT (LRT) (trachea, bronchi, bronchioles, alveoli) resulting in fatal pneumonia in severe cases. Seasonal influenza infections, which are mainly caused by $\mathrm{H} 1 \mathrm{~N} 1$ or H3N2 IAV strains, are responsible for 3-5 million human cases of severe infections and 290,000-650,000 fatal cases annually, most often in young children, the elderly and immunocompromised individuals (2). Pandemic IAV infections affect a broader category of populations and cause atypical and more severe clinical symptoms (1). In aquatic birds, low pathogenic avian influenza viruses (LPAIV) typically cause asymptomatic infections. In poultry, infections with LPAIV can be asymptomatic or provoke low to mild pathophysiological damages to the respiratory, digestive and reproductive systems (resulting in a drop in egg production), while infections with highly pathogenic avian influenza viruses (HPAIV) are characterized by high morbidity and mortality rates. Outbreaks in domestic poultry result in massive culling to control the viral spread and are thus responsible for important economic losses (3).

\section{THE MUCOSAL IMMUNE RESPONSES AGAINST IAV INFECTIONS}

The host RT is not only the initial point of entry and replication of IAV, but also the site of the host immune defenses. Components of the antiviral immune responses are located in the RT lining fluids and in the mucosa-associated lymphoid tissues (MALT) (4). Broadly, in mammals, the MALT of the RT include the nasopharynx-associated lymphoid tissues (NALT) located at the entrance to the nasopharyngeal duct in the URT (Waldeyer's ring in humans), the bronchus-associated lymphoid tissues (BALT) randomly distributed along the LRT but most consistently located at sites of bronchial tree bifurcation, and the draining lymph nodes (DLN). The NALT and BALT are globally composed of organized inductive sites, comprising B cell follicles and interfollicular $\mathrm{T}$ cell areas, as well as effector sites, consisting of the diffuse tissue of the lamina propria. The 
MALT of the RT are overlaid by a surface epithelium made of ciliated cells and various transporter cells located in the follicle-associated epithelium (FAE), such as microfold (M) cells, which transfer the luminal antigens (Ags) to the subepithelial cells (5). MALT are also detected in the avian RT (6). Over the last decade, considerable progress have been done in the understanding of the mechanisms of host mucosal immune responses against IAV (mostly in experimental mouse models of IAV infections).

At the first steps of infection, IAV must face host innate immune molecules with antiviral and/or immunomodulatory activities such as mucins, lectins, complement molecules, natural immunoglobulins (Ig) and antimicrobial peptides (7). Once the virus reaches and infects the epithelium, the local immune cells detect the viral components [also called pathogen-associated molecular patterns (PAMPs)] via pattern recognition receptors (PRRs) including Toll-like receptors (TLRs) (7). These cells produce various cytokines and chemokines involved in the antiviral defense and in the recruitment and activation of innate effector cells, which establish an antiviral program and prime adaptive immune responses.

Neutrophils, natural killer (NK) cells, and monocytes/macrophages interfere with viral replication and spread within the RT, and Ag-presenting cells (APCs) including dendritic cells (DCs) initiate specific adaptive immune responses (7-9). Murine DC subsets can be classified into CD11 $\mathrm{c}^{\text {hi }}$ conventional DCs (cDCs), CD11 $\mathrm{c}^{\mathrm{lo}} \mathrm{B} 220^{+}$plasmacytoid DCs (pDCs) and inflammation-induced $\mathrm{CD}_{11} \mathrm{~b}^{+} \mathrm{Ly}_{6 \mathrm{C}^{+}}$monocytederived DCs (moDCs). In mice, after capturing IAV Ags in the MALT of the RT, respiratory DCs (including CD $103^{+} \mathrm{CD} 11 \mathrm{~b}^{\text {lo }}$ and $\mathrm{CD} 103^{-} \mathrm{CD} 11 \mathrm{~b}^{\text {hi }} \mathrm{cDC}$ subsets) acquire a mature phenotype, transport viral Ags to the DLN and simultaneously process the Ags and present Ag-derived peptides on class I or II major histocompatibility complex (MHC) molecules to naive $\mathrm{CD}^{+}$ and $\mathrm{CD}^{+} \mathrm{T}$ cells, respectively (10-14). Beside migratory cDCs, $\mathrm{CD} 8 \alpha^{+} \mathrm{CD}_{11 b^{-}}$resident $\mathrm{cDCs}$ are also involved in the cross-presentation of IAV Ags to naive $\mathrm{CD}^{+} \mathrm{T}$ cells in the DLN $(10,15)$. Following IAV infection in mice, moDCs accumulate in the RT and they promote immune-induced pathology. However, their complete elimination is detrimental because they facilitate the optimal expansion of effector $\mathrm{CD} 8^{+}$ $\mathrm{T}$ cells in the infected lung (16). Finally, pDCs are a potent source of type I interferons (IFNs), they are involved in the generation of virus-specific antibody (Ab)-secreting cells (ASCs) and they ensure a full-magnitude $\mathrm{CD} 8^{+} \mathrm{T}$ cell response (17-19). Subsets of $\mathrm{CD} 11 \mathrm{c}^{+} \mathrm{CD} 103^{\mathrm{lo}}$ myeloid DCs (mDCs), including $\mathrm{CD} 1 \mathrm{c}^{+} \mathrm{mDC} 1$ and $\mathrm{CD} 141^{+} \mathrm{mDC} 2$ (equivalent to $\mathrm{CD} 103^{-} \mathrm{CD} 11 \mathrm{~b}^{\mathrm{hi}}$ and $\mathrm{CD} 103^{+} \mathrm{CD} 11 \mathrm{~b}^{\text {lo }}$ mouse $\mathrm{CDC}$ subsets, respectively), and pDCs have been identified in the human RT. Studies have suggested that mDCs and pDCs could traffic to the site of IAV infection, but the functionality of the human DC subsets during IAV infection remains largely unknown (20).

Following primary IAV infection in mice, activated $\mathrm{CD} 4^{+}$ and $\mathrm{CD} 8^{+} \mathrm{T}$ cells are detected in the DLN and spleen and the most highly activated and divided cell populations migrate to the airways and notably to the lamina propria of the MALT
$(21,22)$. Activated IAV-specific $\mathrm{CD}^{+} \mathrm{T}$ cells differentiate into effector cytotoxic T lymphocytes (CTLs) which kill virusinfected cells $(23,24)$. Activated IAV-specific $\mathrm{CD}^{+} \mathrm{T}$ cells differentiate into various subsets which present heterogeneous antiviral functionalities and organ location, including $\mathrm{T}$ helper 1 (Th1), Th17 cells, follicular helper $\mathrm{CD} 4^{+} \mathrm{T}$ cells and $\mathrm{CD} 4^{+}$ CTLs (25-27). Studies in humans have mainly examined IAVspecific $\mathrm{T}$ cell responses in the peripheral blood. Secondary responses are generally observed because most adults have encountered IAV Ags multiple times during infections and/or vaccinations. In the absence of specific $\mathrm{Ab}$ responses to newlyemerged IAV, the presence of pre-existing circulating crossreactive $\mathrm{CD} 4^{+}$or $\mathrm{CD}^{+} \mathrm{T}$ cells exhibiting cytotoxic activities correlates with protection against experimentally or naturally mild H1N1 or H3N2 infections in humans (28-30). A recent study showed that rapid and robust IAV-specific $\mathrm{CD} 8^{+} \mathrm{T}$ cell recall responses correlated with early recovery of patients from severe H7N9 disease (30). Most T cell epitopes are highly conserved across IAV and are located on internal proteins such as NP, M1 or polymerase subunits, but they are also present in surface glycoproteins $\mathrm{HA}$ and $\mathrm{NA}$, and in M2 $(9,28,31-33)$.

Follicular helper $\mathrm{CD} 4^{+} \mathrm{T}$ cells support the generation and maintenance of the germinal centers (GCs) in the B cell follicles in cooperation with follicular DCs (34). In GCs, activated B cells, which have received helper signals from cognate $\mathrm{CD} 4^{+} \mathrm{T}$ cells, experience intense proliferation and undergo processes of class switch recombination, somatic hypermutation and affinity selection. GC reactions usually result in the generation of specific long-lived ASCs producing high affinity switched protective Abs and memory B cells. Activated B cells can also participate in early GC-independent reactions, mainly characterized by the rapid generation of low-affinity/specificity ASCs/memory $B$ cells (34). Primary IAV infection in mice results in the development of specific ASCs which present site-specific kinetics and isotype distribution $(35,36)$. Anti-IAV Abs display a variety of functions depending on their isotype, specificity, affinity, concentration and post-translational modifications. Secretory IgA, which are polymeric IgA produced by IgA ASCs in the lamina propria and secreted to the mucosal surface, prevent infection of the epithelial cells via extracellular or intracellular immune exclusion. In addition, polymeric IgA can cross-react with IAV heterovariants (different IAV viruses belonging to the same subtype) or heterosubtypic strains (different IAV viruses belonging to different subtypes) (37). This breadth of reactivity could be linked to the avidity conferred by the polymeric form and/or the effect of the constant heavy chain in modulating the specificity/affinity of the variable regions $(37,38)$. IgA are thus an essential protective front line of defense against highly variable IAV. Whereas, IgG likely play a minor role in supporting secretory IgA in the prevention of IAV infection in the URT, they are crucially involved in the protection of the LRT in mice (39). In humans with low serum hemagglutination-inhibition (HAI) $\mathrm{Ab}$ titers, nasal and serum IgA provide protection against an i.n. experimental H1N1 challenge (40). However, the relative role of mucosal IgA vs. IgG in the (cross)-protection against IAV infection in humans remains poorly characterized. 
IAV-specific Abs generated after an infection are mostly directed against the variable HA1 and, in a lesser extent, against NA (41). Anti-HA Abs mainly interfere with the virus infectivity by preventing virus binding to the sialic acid molecules and entry in epithelial cells. High HAI Ab titers are traditionally considered as the primary correlate of protection against IAV in humans and in animal models (1). Anti-NA Abs prevent the release of newly formed virions. Broadly neutralizing Abs directed against the conserved HA2 can occasionally be elicited in IAV-infected individuals and they interfere with the virus infectivity through several mechanisms (42). Besides neutralizing functions, IAVspecific Abs also exhibit Fc-related effector functions including Ab-dependent cellular cytotoxicity, Ab-dependent phagocytosis and Ab-dependent complement mediated-lysis (43-45). Abs directed against HA2, NA, M2e, NP, and M1 isolated from humans or animals have the potential to mediate protection via Fc-dependent mechanisms (43-45).

The clearance of a primary viral infection results in the establishment of long-lasting memory immune cells detectable in the MALT and in other lymphoid tissues, in the circulation and in the bone marrow. These cells play a critical role in the fight against reinfections. Among influenza-specific memory $\mathrm{T}$ cell populations, lung-resident memory $\mathrm{CD}^{+} \mathrm{T}\left(\mathrm{CD}^{+}\right.$ $\mathrm{T}_{\mathrm{RM}}$ ) cells are crucially involved in the heterosubtypic crossprotection against pulmonary infection in mice $(46,47)$. Virusspecific $\mathrm{CD}^{+} \mathrm{T}_{\mathrm{RM}}$ cells can also be generated in the URT after IAV infection in mice. This cell population can efficiently clear a secondary heterosubtypic IAV infection from the URT and consequently blocks the spread of the virus from the URT to the LRT and the subsequent development of severe pulmonary diseases (48). IAV infection also stimulates the generation of specific lung $\mathrm{CD}^{+} \mathrm{T}_{\mathrm{RM}}$ cells in mice (49) and adoptive transfer experiments demonstrate that these cells have a better protective potential against homologous reinfection than memory $\mathrm{CD} 4^{+} \mathrm{T}$ cells isolated from nonMALT locations (50). IAV-specific lung $\mathrm{CD} 8^{+} \mathrm{T}_{\mathrm{RM}}$ cells are also detected in humans but due to practical and ethical limitations, the implication of mucosal $\mathrm{T}_{\mathrm{RM}}$ cells in the resistance against IAV reinfection in humans is largely unknown $(49,51)$.

Whereas, long-lived ASCs serve as an immediate first line of defense against IAV homologous reinfections by secreting Abs with high specificity/affinity, memory B cells are more specialized to respond to antigenically divergent viruses. In particular, recent studies in mice have shown that GCs in the BALT select cross-reactive $B$ cell repertoire, resulting in the generation of tissue-resident broad reactive memory $\mathrm{B}$ cells (52). The presence of these memory B cell populations at the site of infection facilitates their direct contact with intact influenza virions and could promote a faster production of high-affinity (cross-reactive) Abs through T-independent and PRR-dependent pathways (34). Ab responses induced by natural IAV infections in humans are relatively broad and long-lived (41). However, further investigations are needed to localize and identify cell subsets involved in these memory cross-reactive humoral responses (41).

\section{PROBLEMATIC ISSUES RELATED TO CURRENT IAV VACCINES}

Vaccination remains the most efficient and cost-effective means to protect human and animal populations against IAV (1). Most influenza vaccines available on the market are inactivated influenza A virus (IIV) vaccines administered via the parenteral route. Three types of IIV vaccines exist, namely whole inactivated virus (WIV) vaccines consisting of formaldehyde- or $\beta$ propiolactone-inactivated whole virion, split virus vaccines, and subunit vaccines. In split virus vaccines, the virus envelope is broken by diethyl ether or detergent treatment that disrupts the particulate organization and exposes all viral proteins, while subunit vaccines consist of surface proteins HA and NA separated from the nucleocapsid and lipids. While WIV preparations are commonly used in human pre-pandemic vaccines and in poultry, current human seasonal vaccines are mainly split virus or subunit vaccines. Current IIV vaccines predominantly induce virus-specific $\mathrm{Ab}$ responses directed against HA1 and do not stimulate efficient cellular immune responses. Thus, the efficiency of these vaccines is restricted to the protection against homologous/antigenically similar strains (1).

Beside IIV vaccines, live-attenuated influenza virus (LAIV) vaccines administered via the intranasal (i.n.) route are also available in humans. LAIV vaccines are composed of coldadapted virus strain(s) restricted to the URT and causing only mild symptoms (1). LAIV and IIV vaccines are equally effective in adults, while studies have concluded that LAIV vaccines are more efficient in children, generating broader and longer-lived immune responses. Indeed, LAIV vaccines mimic the natural route of infection and consequently induce stronger mucosal IgA and broader $\mathrm{T}$ cell-mediated immune responses than IIV vaccines (1). A recent study in mice comparing two different licensed influenza vaccines showed that, in contrast to IIV vaccines injected parenterally, i.n. LAIV vaccines elicited the generation of lung $\mathrm{T}_{\mathrm{RM}}$ cells, conferring long-term protection against various non-vaccine strains (53). Despite very promising results, some drawbacks have been associated with the use of LAIV vaccines (1). A suboptimal protection of LAIV vaccines in children against the $2009 \mathrm{H} 1 \mathrm{~N} 1$ pandemic virus has been reported in the USA, which might be due to an impaired viral replication in the URT, resulting in a decreased stimulation of the host (mucosal) immune system. Also, for safety concerns, LAIV vaccines are contraindicated in children $<2$ years old and in immunocompromised individuals. Finally, the virus strain(s) composing LAIV vaccines could theoretically undergo genetic reassortments with circulating wild type viruses (1).

Thus, due to its ability to generate broadly reactive and longterm protective immune responses at the front line of virus entry, vaccination via the mucosal route is an appropriate strategy for the prevention and control of IAV infections. However, current mucosal vaccines need to be reformulated into safer, more refined and immunogenic preparations. In order to counteract the high variability of IAV, an active area of research area focusing on developing subunit vaccine candidates containing conserved surface or internal protein(s)/epitope(s) of the virus has garnered 
much interest. These "universal" vaccines aim at generating cross-reactive Abs and/or cross-reactive $\mathrm{T}$ cell responses (1). Alongside WIV, split or protein subunit vaccines, nucleic acidbased subunit vaccines induce vaccine Ag production in the host itself and can engage both humoral and cell-mediated immune responses $(1,31)$. Pre-clinical and clinical studies have shown that nucleic acid-based IAV vaccines can be efficiency delivered by live viral vectors through parenteral or mucosal routes and viral-vectored vaccines are commercially available for the control of avian IAV in poultry $(54,55)$. Nucleic-acid based vaccines delivered via non-lived vectors mimic infection or immunization with live microorganisms while being non-infectious, egg/cellfree manufacturable (ensuring a rapid, cost-effective and scalable production) and they do not have to address the challenge of preexisting or induced anti-vector immunity which could prevent repeated immunizations (56-58).

\section{ADJUVANTS/DELIVERY SYSTEMS FOR INTRANASAL VACCINATION AGAINST IAV INFECTIONS}

The goal of vaccination is to generate potent and longterm protective immune responses against infections. Unlike attenuated live vaccines, inactivated vaccines (especially purified or recombinant subunit vaccines) usually require additional compounds to be effective. The role of an adjuvant is to increase vaccine efficacy by modeling the quality and the quantity of the host immune responses. In particular, the adjuvant can affect the magnitude, breadth, specificity, affinity, kinetics, longevity, and composition of the immune responses (59-61). The incorporation of adjuvant(s) into a vaccine formulation can decrease the amount of the vaccine $\mathrm{Ag}(\mathrm{s})$ and the number of doses required to induce protective immunity, and enhance vaccine efficacy in special populations (the elderly, neonates/infants, immunocompromised individuals) (59-61). Adjuvants were formerly classified as delivery systems, which function as vehicles to which the vaccine $\operatorname{Ag}(\mathrm{s})$ can be associated, and immune potentiators. However, there is now evidence that some delivery systems also exhibit immunostimulatory properties. Adjuvants exert their effect through various mechanisms including the formation of a depot at the site of injection, which guarantees a slow release of the vaccine $\mathrm{Ag}(\mathrm{s})$ and a constant stimulation of the host immunity (depot effect), the recruitment of immune cells, the activation of innate immune receptors, the enhancement of Ag uptake by APCs and the activation and the maturation of APCs (59-61). By targeting innate immune cell subsets and by generating a particular cytokine milieu (Th1, Th2, and/or Th17 for example), adjuvants can shape the nature of the subsequent adaptive immune responses to produce the most effective and protective reaction against a given pathogen.

Despite the relevance of the mucosal route of vaccination in the fight against respiratory pathogens, this method of administration faces several hurdles (62). In addition to encountering a tolerogenic mucosal environment, vaccine $\mathrm{Ag}(\mathrm{s})$ must resist degradation caused by the harsh mucosal environment characterized by the presence of proteases, nucleases and low $\mathrm{pH}$. In addition, the $\mathrm{Ag}(\mathrm{s})$ is (are) likely to be diluted in the mucosal fluids and swept away by the mucociliary clearance. Consequently, mucosal vaccines have to be carefully formulated with adjuvants in order to breach the host mucosal barriers and allow the vaccine $\mathrm{Ag}(\mathrm{s})$ to reach and stimulate the cells of the underlying MALT (62). Mucosal adjuvants should ensure the integrity and the stability of vaccine $\mathrm{Ag}(\mathrm{s})$, exhibit mucoadhesive properties and allow Ag uptake by epithelial cells and/or $\mathrm{M}$ cells. The rationale and challenges for the development of IAV mucosal vaccines are presented in Figure 1.

In this section, we have compiled the delivery/adjuvant systems tested for i.n. administration of IIV vaccines with a special emphasis on experimental animal studies (see Supplemental Table 1). We have focused our work on nonreplicative delivery/adjuvant systems (excluding viral and bacterial vectors).

\section{Bacterial Enterotoxins and Derivatives}

The most potent and studied mucosal adjuvants in pre-clinical studies are cholera toxin (CT), the closely related Escherichia coli heat-labile toxin (LT) and their derivatives (detoxified/nontoxic holotoxins, isolated subunits). CT and LT holotoxins are composed of a pentameric B subunit, which binds to GM1ganglioside receptors ubiquitously present on the surface of most nucleated cells, non-covalently associated with an A subunit. The A1 portion of the A subunit enzymatically ADP-ribosylates the $\alpha$ subunit of the GTP-binding protein Gs (Gs $\alpha$ ), causing a dramatic elevation of intracellular cyclic AMP responsible for the efflux of ions and water from the targeted cells associated with watery diarrhea. The mucosal adjuvant properties of enterotoxins rely on a better accessibility of the co-administered $\mathrm{Ag}(\mathrm{s})$ to the cells of the MALT, which is related to an increased permeability of the mucosal barriers and/or to an enhancement of the recruitment and activation of local APCs (63).

Experimental studies in mice have demonstrated that these holotoxins are potent immunostimulators for mucosal IIV vaccines $(64,65)$. For example, the addition of CT to H1N1 WIV vaccine administered to mice via the i.n. route enhanced the magnitude of the serum and mucosal $\mathrm{Ab}$ responses directed against homologous, H1N1 heterovariant and H3N2 heterosubtypic viruses (65). CT increased the breadth of protection of the vaccine and all mice receiving the adjuvanted formulation survived the i.n. $\mathrm{H} 3 \mathrm{~N} 2$ heterosubtypic challenge (65). The adjuvant also stimulated the generation of long-lived ASCs lodged in the bone marrow that secreted IgA and IgG reactive against the homologous and the $\mathrm{H} 3 \mathrm{~N} 2$ heterosubtypic viruses (65). Holotoxins engineered into less toxic molecules, through site-directed mutations of the enzymatically active A subunit, and administered with influenza WIV or subunit vaccines also retained adjuvant functions $(66,67)$. However, the i.n. delivery of vaccines formulated with native or even detoxified LT in humans resulted in the development of facial paralysis (Bell's palsy) in some individuals, stopping further clinical use of enterotoxin-based adjuvants (63).

Enterotoxins lacking B subunit are good alternatives to i.n. holotoxin-based adjuvants because they keep strong adjuvant 


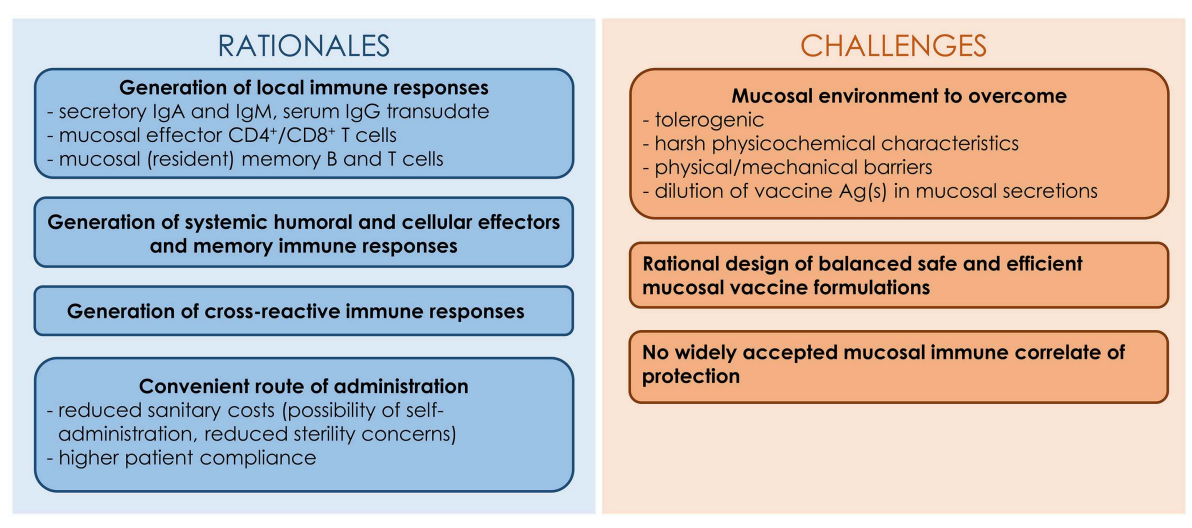

FIGURE 1 | Rationales and challenges for the development of IAV mucosal vaccines.

functions without toxic side effects $(68,69)$. For example, a study in mice evaluated the ability of the A1 subunit of the CT (CTA1) to potentiate the immunogenicity of a subunit vaccine composed of a portion of $\mathrm{HA} 2$ of a $\mathrm{H} 5 \mathrm{~N} 1$ virus $\left[\mathrm{HA}_{\mathrm{H} 5 \mathrm{~N} 1(15-137)}\right]$ fused with an M2 consensus sequence spanning the residues of the extracellular and cytoplasmic domains of M2 from H1N1, H5N1

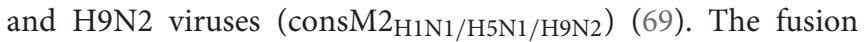

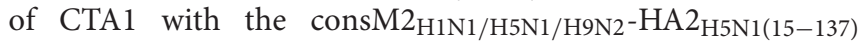
chimeric protein increased the magnitude and persistence of M2/HA2-specific serum and mucosal humoral responses and M2/HA2-specific cellular immune responses in the spleen. Mice immunized with the CTA1-adjuvanted vaccine showed a better resistance to an i.n. challenge with divergent subtypes of IAV (H1N1, H5N1, H5N2, H7N3, H9N2) than mice immunized with the vaccine without CTA1 (69).

The chimeric protein composed of CTA1 fused with a synthetic dimer of the Ig binding D domain (DD) from Staphylococcus aureus protein A (CTA1-DD) is also a promising adjuvant for next generation mucosal vaccines against IAV. The DD portion of the molecule targets the $\mathrm{B}$ cell receptor (BCR) as well as the complement receptor CD21 on follicular DCs, resulting in an enhancement of GC formation $(63,70)$. In addition, experiments in mice and non-human primates have proven the safety of CTA1-DD (63). In pre-clinical tests, CTA1-DD increased the immunogenicity and/or the protective potential of various influenza subunit vaccines administered intranasally, either in fusion with M2e epitopes $(71,72)$, admixed with virus-like particles (VLPs) exposing M2e epitopes (73), or incorporated into lipid-based particles with IAV Ags (70). For example, the incorporation of CTA1-DD into immune stimulating complexes (ISCOMs) containing H1N1 HA and NA boosted the specific serum and mucosal humoral responses as well as type 1/type 2cell-mediated immune responses in the spleen in mice. The immunostimulatory effects of CTA1-DD depended both on the enzymatic activity of the toxin and on B cell targeting (70). These encouraging results make CTA1-DD a very attractive adjuvant for mucosal anti-IAV vaccines for clinical or veterinary use (63).

\section{Flagellin}

Flagellin, the primary structural component of the bacterial flagellum, is another promising mucosal adjuvant for antiIAV vaccines. Flagellin targets TLR5 expressed at the surface of various cells including airway epithelial cells, DCs and lymphocytes, as well as cytosolic detectors of the NAIP (nucleotide oligomerization domain-like receptor (NLR) family, apoptosis inhibitory protein) family proteins (74). The mucosal adjuvant properties of flagellin rely on the acceleration of the transepithelial transport of the co-administered $\mathrm{Ag}(\mathrm{s})$ by the FAE and on the stimulation of the migration of subepithelial DCs into the FAE (75).

The i.n. co-administration of recombinant flagellin derived from Vibrio vulnificus or Salmonella typhimurium with H1N1 WIV vaccine or a trivalent split vaccine composed of two IAVs (H1N1 and H3N2) and an influenza B strain (IBV) boosted the immunogenicity of the vaccine in mice $(76,77)$. In particular, the adjuvant increased the serum and/or mucosal $\mathrm{Ab}$ responses and elicited systemic type 2-biased-cell-mediated immune responses $(76,77)$. The incorporation of flagellin into the vaccine preparation enhanced protection of mice against a subsequent i.n. homologous challenge $(76,77)$. The adjuvant exhibited a safer profile than enterotoxins because it did not accumulate in the central nervous system (77).

Flagellin is also a potent mucosal adjuvant for influenza subunit vaccines (78-80). The association of flagellin with IAV epitopes, as free fusion proteins or as membrane-anchored form into influenza VLPs, significantly increased the immunogenicity and the (cross)-protective potential of these vaccines against IAV in mice (78-80). For example, the membrane incorporation of flagellin into H1N1 (HA/M1) VLPs enhanced serum and mucosal $\mathrm{Ab}$ titers and elicited systemic type 1/type 2-cell-mediated immune responses specifically directed against the homologous H1N1 strain (78). Moreover, the addition of flagellin to the vaccine formulation induced higher cross-neutralizing Ab titers to a $\mathrm{H} 3 \mathrm{~N} 2$ virus detectable in the serum and in the lung lavage (78). Accordingly, mice immunized with the adjuvanted vaccine were all resistant to the i.n. H3N2 heterosubtypic challenge in contrast to the unadjuvanted group (78). Another in vitro study 
showed that the conjugation of flagellin on the surface of gold nanoparticles bearing a recombinant trimeric HA derived from a $\mathrm{H} 3 \mathrm{~N} 2$ virus stimulated the uptake of the vaccine by murine bone marrow-derived DCs and promoted the activation and the maturation of the cells (81). Mice intranasally immunized with such flagellin-adjuvanted vaccine developed higher influenzaspecific immune responses and showed a better resistance against an i.n. homologous challenge $(81,82)$.

Interestingly, flagellin also significantly boosted the immunogenicity and the protective capacity of IIV vaccines in chickens, and notably when it was admixed with H5N2 WIV vaccine or fused with an epitope located in HA1 of a H7N9 virus $(83,84)$.

Clinical trials have proven the safety and the immunogenicity of flagellin-adjuvanted influenza subunit vaccines when administered via the parenteral route $(85,86)$. Further clinical investigations on the efficacy of this adjuvant in mucosal anti-IAV vaccination are needed.

\section{Proteosome-Based Adjuvants}

Proteosomes and Protollin are two other potent mucosal immunoactivators. Proteosomes are nanoparticles composed of a mixture of outer membrane proteins and traces of lipooligosaccharides derived from Neisseria meningitidis and Protollin consist of proteosomes complexed with Shigella flexneri lipopolysaccharides. These adjuvants stimulate Ag uptake by the FAE and cells of the MALT, and promote the maturation and activation of APCs via the engagement of various PRRs (87). Preclinical tests in mice concluded that proteosomes and Protollin were safe and strong mucosal adjuvants for split $(88,89)$ or subunit $(89,90)$ IAV vaccines. In addition, recent clinical trials showed that an i.n. proteosome-adjuvanted trivalent split vaccine was safe, immunogenic, and effective in healthy adults (91). The vaccination protected subjects against illness following an experimental $\mathrm{H} 3 \mathrm{~N} 2$ homologous challenge, and the protection was correlated with pre-challenge specific serum HAI Ab and nasal IgA titers (91). Thus, this class of adjuvants deserves to be harnessed for the development of mucosal anti-IAV vaccination.

\section{Bacterial-Derived Vesicles Bacterium-Like Particles}

Bacterium-like particles, also known as Gram-positive enhancer matrix, are non-living microparticles derived from nonpathogenic food-grade bacteria Lactococcus lactis, and consist of bacterial-shaped peptidoglycan spheres deprived of intact surface proteins and intracellular content. The immunostimulating properties of bacterium-like particles are related to their ability to activate and stimulate the maturation of APCs (92). In mice, bacterium-like particles admixed with $\mathrm{H} 1 \mathrm{~N} 1$ or $\mathrm{H} 3 \mathrm{~N} 2$ split vaccine enhanced serum and mucosal humoral responses as well as systemic type 1-biased cellular responses directed against the vaccine strain $(93,94)$. Moreover, the presence of the particles in the vaccine formulation significantly increased the level of protection of mice against an i.n. homologous challenge (94). The adjuvanticity of bacterium-like particles was suggested to be TLR2-dependent (95). Successful pre-clinical tests were also reported using bacterium-like particles non-covalently coupled with purified influenza epitopes (HA, M2e or NP) (92).

Finally, a phase I clinical trial has proven the safety and the immunogenicity of i.n. vaccine formulations composed of seasonal influenza trivalent split vaccine mixed with bacteriumlike particles (92). In particular, the adjuvant boosted the specific serum HAI Ab and nasal IgA titers and increased the frequency of IFN- $\gamma$-producing peripheral blood mononuclear cells (92).

\section{Outer Membrane Vesicles}

Outer membrane vesicles are nanosized vesicles naturally produced by Gram-negative bacteria (96). They are composed of various PAMPs such as lipopolysaccharides, lipoproteins, peptidoglycan, flagellin monomers and nucleic acids, and they can stimulate the activation of respiratory $\mathrm{CD}_{103}{ }^{+} \mathrm{DCs}(96)$. The incorporation of outer membrane vesicles into a trivalent split vaccine boosted influenza-specific serum IgG and HAI $\mathrm{Ab}$ titers as well as IgG and IgA titers in the LRT in mice receiving the vaccine formulation via the i.n. route (96). In addition, the adjuvant stimulated type 1-cell-mediated immune responses in the lungs and spleen (96). All mice survived an i.n. homologous challenge and were significantly more resistant to $\mathrm{H} 1 \mathrm{~N} 1$ heterovariant or $\mathrm{H} 5 \mathrm{~N} 2$ heterosubtypic challenges than mice immunized with the unadjuvanted vaccine (96).

\section{Lipid-Based Adjuvants Liposomes}

Liposomes are self-assembling bi- or multi-layered lipid vesicles with an aqueous core ranging from $10 \mathrm{~nm}$ to several $\mu \mathrm{m}$ in diameter (97). Various categories of lipids can be incorporated into liposomes such as phospholipids, sterols (cholesterol) or sphingolipids. Liposomes are common vaccine delivery vehicles because they protect the associated $\mathrm{Ag}(\mathrm{s})$ from the degradation or neutralization, they exhibit Ag depot effect and they are uptaken by APCs (97). Several physicochemical features of the liposomal formulations determine their immunomodulatory potentials including the lipid characteristics and the lipid composition of the liposomes (97). For example, the length and the degree of saturation of the hydrophobic tail of the lipids govern the fluidity/permeability and thus the stability of the particles. Cholesterol commonly modulates the stability of liposomes. In addition, the hydrophilic headgroups of the lipids determine the surface charge and, by extension, the ability of the liposomes to adhere/penetrate epithelial surfaces (in the context of a mucosal vaccination). The localization of the vaccine $\mathrm{Ag}(\mathrm{s})$ in the preparation, e.g., admixed with the liposomes, attached to the surface of the liposomes or encapsulated in the aqueous core of the liposomes, also influences the Ag-specific immune responses. Finally, the adjuvanticity of the liposomes can be improved by the incorporation of muco-adhesive/muco-penetrating polymers or PRR ligands into the formulation (97).

Both pre-clinical and clinical trials have demonstrated the remarkable adjuvant potential of liposomes in parenteral vaccination against IAV. Encouraging results have also been obtained in the context of mucosal vaccination $(97,98)$. Early studies showed that anionic dimyristoyl phosphatidylcholine (DMPC)/dimyristoyl phosphatidylglycerol (DMPG) liposomes 
facilitated the association and uptake of co-administered molecules by macrophages in vitro (99). The co-encapsulation of H1N1 split vaccine in DMPC/DMPG liposomes with synthetic oligodeoxynucleotides composed of unmethylated $\mathrm{CpG}$ motifs (CpG-ODN, TLR9 ligands) boosted the magnitude of virusspecific IgG and IgA titers in the URT and LRT and induced systemic type 1-biased cell-mediated immune responses in intranasally immunized mice (99). In addition, mice receiving the liposome-formulated vaccine showed reduced lung viral loads after an i.n. heterovariant challenge (99). CAF01, another liposomal preparation containing the monocationic lipid dimethyldioctadecylammonium bromide (DDAB) and the immunostimulator $\alpha, \alpha^{\prime}$-trehalose 6,6'-dibehenate was effectively uptaken by murine bone marrow-derived DCs in vitro and stimulated the maturation of the cells (100). By using the human bronchial epithelial Calu- 3 cell culture model, CAF01 was shown to enhance the transport of co-administered Ag through the mucus layer and across the epithelial cells (101). Accordingly, in mice, the i.n. administration of CAF01 with H3N2 (100) or seasonal trivalent (101) split vaccines remarkably strengthened virus-specific mucosal and systemic immune responses. Finally, polycationic liposomes composed of ceramide carbamoylspermine (CCS) sphingolipids complexed with cholesterol enhanced the efficacy of H1N1 monovalent or seasonal trivalent split vaccines $(102,103)$. Mice intranasally immunized with the vaccine adjuvanted with CCS/cholesterol liposomes showed an increase in serum and mucosal HAI Ab titers directed against homologous or heterovariant viruses $(102,103)$. In addition the adjuvant increased type 1-cell-mediated immunity in the spleen $(102,103)$. Finally, the animals exhibited a better resistance to i.n. homologous or heterovariant challenges than mice immunized with the unadjuvanted vaccine (103). CCS/cholesterol liposomes presented similar adjuvant effects to CT, while having a better safety profile (103). Interestingly, CCS/cholesterol liposomes had stronger adjuvant properties than anionic DMPC/DMPG or monocationic dioleoyl-3- trimethylammoniumpropane (DOTAP)/cholesterol liposomes, which could be linked to an extended retention time of influenza Ags in the nasal cavity and in the lungs $(102,103)$. Finally, CCS/cholesterol liposomes also enhanced the immunogenicity of $\mathrm{H} 1 \mathrm{~N} 1$ split vaccine in aged mice, albeit with lower specific serum and mucosal Ab titers than those observed in adult mice (103). Clinical trials with i.n. liposome-based influenza split vaccine have been reported $(97,104)$.

Some studies have demonstrated that liposomes are also potent mucosal carriers for influenza subunit vaccines in mice (105-107). For example, an M2e consensus sequence incorporated into the lipid bilayer of liposomes composed of a mixture of phospholipids, cholesterol and monophosphoryl lipid A (MPLA) generated M2e-specific serum IgG and systemic type 1/type 2-cell mediated immune responses in mice vaccinated subcutaneously and intranasally (107). The animals were also significantly protected against an i.n. homologous challenge (107). Interestingly, the M2e-specific serum $\mathrm{Ab}$ response inhibited in vitro viral cell lysis by various IAV subtypes including H2N2, H3N2, H6N2, H5N9, and H1N1 IAV, highlighting the potential of liposomes as mucosal delivery platforms for broadly-protective vaccines against IAV (107).

Preclinical and clinical studies have also concluded that cationic liposomes are attractive delivery platforms for nucleicacid based IAV vaccines. Cationic lipids are known to efficiently complex the nucleic acids, facilitate cellular uptake and allow endosomal escape of nucleic acids into the host cytoplasm (57). The encapsulation of plasmid DNA encoding a HA protein derived from a H1N1 strain into cationic liposomes administered to mice via the i.n. route boosted the immunogenicity of the vaccine and all animals survived a subsequent i.n. homologous challenge unlike mice vaccinated with the naked plasmid DNA (105). Over the past decade, major technological advances have enabled mRNA vaccines to become promising candidates against IAV infections $(57,58,108)$. In contrast to plasmid DNA, mRNAbased vaccines are delivered directly to the cytoplasmic site of function, eliminating the potential risk of integration into the host chromosome. The engineering of mRNA sequence and the development of separation and/or purification techniques can increase mRNA translation and stability and modulate its inherent immunogenicity $(57,58,108)$. In addition, several delivery systems have been explored to improve the efficiency of mRNA vaccines, including liposomes (109). These carriers are usually composed of an ionizable cationic lipid, cholesterol, phospholipids, and lipid-linked polyethylene glycol which increases the half-life of the formulation (109). Liposomeencapsulated mRNA vaccines encoding IAV Ags such as HA, NP and/or M1 administered via intramuscular or intradermal routes generated significant $\mathrm{B}$ and/or $\mathrm{T}$ cell immune responses and conferred protection against i.n. homologous or heterosubtypic IAV challenge in various experimental animal models including mice, ferrets and/or non-human primates (110-112). A recent clinical study showed that mRNA vaccine encoding a HA protein derived from $\mathrm{H} 10 \mathrm{~N} 8$ or H7N9 viruses formulated with liposomes and administered via the intramuscular route was safe and immunogenic (111). While lipid nanoparticles are potent delivery systems for nasal mRNA vaccines in the context of anti-tumor vaccination (113), additional studies are needed to evaluate the efficacy of such carriers in mucosal vaccination against IAV.

\section{Virosomes}

Virosomes are a special category of liposomes which are composed of purified or synthetic lipids and viral envelope proteins such as HA and NA ("influenza virosomes") (98). Influenza virosomes are commercially available, safe and efficient vaccine platforms for parenteral vaccination in humans. However, there have been no new i.n. formulations on the market since the withdrawal of LT-adjuvanted virosomal vaccines (98). Among various strategies to potentiate the immunogenicity of i.n. virosomal vaccines against IAV, the incorporation of cyclic di-nucleotides (CDN) (see section Nucleotide-Based Adjuvants) has proven to be valuable in pre-clinical tests (114). Mice immunized with $\mathrm{H} 5 \mathrm{~N} 1$ virosomes admixed with cyclicdi-adenosine monophosphate (c-di-AMP) developed stronger, broader and more persistent anti-IAV immune responses than mice immunized with the unadjuvanted virosomes (114). In 
particular, the animals showed higher mucosal IgA titers and higher serum HAI Ab titers reactive against homologous and heterovariant viruses. Moreover, the adjuvant increased the frequency of long-lived IgG ASCs lodged in the bone marrow. Mice also exhibited stronger systemic type 1/type 2/type 17 cellular immune responses, including higher frequency of multifunctional influenza-specific $\mathrm{CD} 4^{+} \mathrm{T}$ cells in the spleen (114). Finally c-di-AMP promoted protection of mice against i.n. homologous challenge (114). Clinical trials with virosome-based influenza vaccines administered via the nasal route are ongoing $(98,115)$.

\section{Immune Stimulating Complexes (ISCOMs)/ Immune Stimulating Complex Matrix ISCOMATRIX}

ISCOMs are negatively-charged pentagonal dodecahedrons with a ring-like stable structure about $40 \mathrm{~nm}$ in diameter which are spontaneously formed after mixing the vaccine $\mathrm{Ag}(\mathrm{s})$ with cholesterol, phospholipids and saponins from the Quillaja saponaria Molina tree (QuilA) which are potent immunostimulators (98). The corresponding structure without incorporated vaccine $\mathrm{Ag}$ is called ISCOMATRIX. ISCOM/ISCOMATRIX adjuvants are efficiently processed by various APCs and are thus widely-exploited vaccine delivery systems. The high affinity between saponins and cholesterol ensures the stability of the adjuvant (116). Vaccine formulations with more refined fractions of QuilA saponins have been developed in order to increase the safety and the tolerogenicity of the adjuvant (117). Pre-clinical studies have shown that this category of adjuvants markedly increases the immunogenicity and the protective potential of i.n. split or subunit IIV vaccines, with the viral Ags incorporated into ISCOMs or simply admixed with the ISCOMATRIX $(70,117,118)$. Notably, mice intranasally immunized with an ISCOM/ISCOMATRIX-formulated H1N1 split vaccine presented higher nasal/lung IgA and serum IgG titers than mice vaccinated with the non-adjuvanted vaccine (118). Two other studies indicated that mice receiving envelope Ags derived from H1N1 virus in ISCOM/ISCOMATRIX formulations via the i.n. route exhibited stronger serum and mucosal $\mathrm{Ab}$ responses as well as higher systemic type 1/type 2 cellular immunity than mice receiving the free Ags $(70,117)$. The immunogenicity of ISCOM/ISCOMATRIX preparations can be boosted by the inclusion of immunostimulators (117). The addition of CTA1-DD into ISCOM/ISCOMATRIX formulations resulted in generating attractive and versatile mucosal delivery platforms for IAV Ags by targeting both DCs and B cells (117). Of note, a CTA1-3M2e-DD/ISCOM preparation could be kept 1 year at $4^{\circ} \mathrm{C}$ or as freeze-dried powder without altering the immunogenicity of the vaccine (117). ISCOMs are thus promising adjuvant for the development of cold chain-independent vaccines. Finally we can mention that ISCOMATRIX is also a suitable mucosal adjuvant for H5N1 WIV vaccines in chickens (119). Clinical trials with i.n. ISCOM-based influenza vaccines are currently under way $(98,116)$.

\section{Other Lipid-Based Adjuvants}

Surfacten is a modified bovine pulmonary surfactant forming unilamellar vesicles about $300-1,000 \mathrm{~nm}$, which is widely used in premature babies with respiratory distress syndrome without significant adverse effects. It also displays potent mucosal adjuvant properties toward influenza split vaccines in mice (120, 121). More precisely, Surfacten enhanced the uptake of influenza Ags by bone marrow-derived DCs in vitro, and increased the distribution and the retention time of influenza Ags as well as the maturation of $\mathrm{CD}_{11 \mathrm{c}^{+}}$cells in the nasal cavity in vivo $(120,121)$. The adjuvant boosted both virus-specific local and systemic humoral and cell-mediated immunity and enhanced the protection of mice against i.n. homologous or heterovariant challenge $(120,121)$. Three major lipids, namely dipalmitoyl phosphatidylcholine (DPPC), phosphatidylglycerol (PG) and palmitate, and the surfactant protein C (SP-C) play an essential role in the adjuvant properties of Surfacten (121).

SF-10, a synthetic surfactant composed of DPPC/PG/palmitate lipids, a cationic SP-C-related peptide and a mucoadhesive carboxyvinyl polymer (CVP) is also a safe and effective delivery vehicle for IAV split vaccines in mice and non-human primates (122-124). In vivo experiments in mice showed that SF-10 stimulated the delivery of co-administered Ags to epithelial cells and APCs localized in the NALT, including $\mathrm{CD}^{+} \mathrm{CD} 11 \mathrm{c}^{+}$DCs known to be involved in the cross-priming of $\mathrm{CD}^{+} \mathrm{T}$ cells (123). Accordingly, the i.n. administration of SF-10 with H1N1 split vaccine strengthened virus-specific serum and mucosal $\mathrm{Ab}$ responses and $\mathrm{T}$ cell-mediated responses $(122,123)$. In particular, mice immunized with the SF-10supplemented vaccine showed higher frequency of IFN- $\gamma$ - and IL-4-secreting lymphocytes in the NALT and CD8 ${ }^{+}$CTLs in the spleen $(122,123)$. These mice were also more resistant to an i.n. heterovariant challenge than those immunized with the unadjuvanted vaccine, and the protection was significantly reduced after depletion of $\mathrm{CD}^{+}$or $\mathrm{CD}^{+} \mathrm{T}$ cells (123). The resistance was associated with higher cytolysis activities in the lungs and DLN in the early phase of infection (123). Finally, young cynomolgus monkeys immunized with H1N1 split vaccine admixed with SF-10 via the i.n. route developed humoral immune responses composed of nasal Abs which cross-reacted against $\mathrm{H} 1 \mathrm{~N} 1$ heterovariant and $\mathrm{H} 3 \mathrm{~N} 2$ heterosubtypic viruses, and serum HAI Abs (124). Moreover, the animals exhibited influenza-specific immunological memory (124). SF-10 is thus a very promising adjuvant for i.n. influenza vaccines intended for humans, and in particular for young children.

Endocine is an anionic adjuvant based on mono-olein and oleic acid lipids found ubiquitously in the human body. Clinical vaccine studies against diphtheria and the human immunodeficiency virus (HIV) have proven that Endocine is a safe and well-tolerated i.n. adjuvant (125). Several studies have demonstrated that it is also a promising mucosal adjuvant for IAV vaccines (125-127). The addition of Endocine to an i.n. split trivalent vaccine enhanced both local and systemic crossreactive humoral and cell-mediated immune responses in adult mice (126). The adjuvant is also a potent immunoenhancer in immunocompromised mice (127). Aged mice immunized with an Endocine-adjuvanted H1N1 split vaccine exhibited increases in influenza-specific serum IgG and HAI Ab titers and lung IgG and IgA titers (127). Finally, ferrets intranasally immunized with H1N1 split or WIV vaccine formulated with Endocine 
developed serum HAI and neutralizing Ab responses against homologous and H1N1 heterovariant viruses (125). Animals challenged intratracheally with the homologous strain were significantly protected from virus replication in the URT and LRT (125). Further (pre-)clinical investigations on the use of Endocine in i.n. IIV vaccine formulations are warranted.

Among other lipid-based adjuvants which have proven to be successful in mucosal vaccination against IAV in (pre)clinical tests, we can cite the oil-in-water nanoemulsion $\mathrm{W}_{80} 5 \mathrm{EC}$, consisting of a highly refined soybean oil combined with nonionic and cationic surfactants and ethanol. In vitro and in vivo studies in mice suggested that the adjuvanticity of $\mathrm{W}_{80} 5 \mathrm{EC}$ was related to its ability to increase the uptake of the antigenic payload by nasal epithelial cells, facilitating the subsequent engulfment of Ag-primed apoptotic epithelial cells by DCs, followed by activation and migration of DCs to the DLN (128, 129). The adjuvant activity of $\mathrm{W}_{80} 5 \mathrm{EC}$ is related, at least in part, to TLR2/TLR4 activation $(130,131)$. Accordingly, $W_{80} 5 E C$ enhanced the immunogenicity and the protective potential of i.n. H1N1 WIV vaccine in mice (132). The i.n. administration of $\mathrm{W}_{80} 5 \mathrm{EC}$ with a seasonal IAV split vaccine also boosted serum humoral responses directed against homologous and heterovariant viruses in ferrets (133). Finally, a clinical study conducted with healthy human adults revealed that $\mathrm{W}_{80} 5 \mathrm{EC}$ was safe, well-tolerated and boosted the influenza-specific serum and mucosal $\mathrm{Ab}$ responses when combined intranasally with a seasonal trivalent split vaccine (134). A recent study mentioned that an mRNA vaccine expressing a HA protein derived from a H1N1 virus formulated with an oil-in-water cationic nanoemulsion elicited broad and protective immune responses against homologous and heterologous challenge when delivered intramuscularly to mice or ferrets (135). However, the efficiency of such formulations in the context of a mucosal immunization remains to be evaluated.

\section{Virus-Like Particles (VLPs)}

VLPs are multimeric cage-like structures consisting of selfassembled structural viral proteins around a hollow interior space devoid of viral genetic material. VLPs mimic virions in shape, size, and molecular organization while being non-replicating and non-infectious $(136,137)$. VLPs can interact with APCs of the innate immunity. In addition, VLPs display highly repetitive epitopes and can stimulate B cell responses by BCR crosslinking (136).

Several pre-clinical studies have demonstrated that VLPs are potent i.n. delivery vehicles for IAV epitopes $(79,138)$. Influenza VLP vaccines are mostly produced by the recombinant baculovirus/insect cell expression system and consist of selfassembled M1 molecules surrounded by the lipid membrane derived from the insect cells where various combinations of influenza epitopes are anchored, including HA, NA and/or M2(e) $(136,137)$. Most of influenza VLPs fully protect animals against an i.n. homologous challenge, and several studies have reported their efficacy against heterologous viruses as well $(136,137)$. For example, the i.n. immunization of mice with VLPs incorporating H1N1 HA, NA and M1 [(HA/NA/M1) VLPs] induced the generation of IgG and IgA in the serum, the URT and LRT, which cross-reacted with a H5N1 virus (139). Mice were fully protected against a subsequent i.n. homologous challenge and exhibited a partial but significant resistance to a challenge with the $\mathrm{H} 5 \mathrm{~N} 1$ virus (139). In contrast, all mice receiving the vaccine via the intramuscular route succumbed to the heterosubtypic challenge. Ferrets intranasally immunized with the same vaccine survived the H5N1 challenge and displayed reduced nasal viral loads, unlike animals vaccinated via the parenteral route (139).

Numerous pre-clinical studies have proven that i.n. influenza VLP vaccines elicit long-lasting (cross-) protective immune responses (140-142). For example, long-lived IgA/IgG ASCs and memory $\mathrm{CD} 4^{+} / \mathrm{CD}^{+} \mathrm{T}$ cells were detected in the bone marrow and in the spleen of mice immunized with H1N1 (HA/M1) VLPs, respectively (140). All mice survived an i.n. homologous or heterovariant challenge up to 5 months post-immunization (140). Also, a recent study showed that the i.n. administration of a mixture of (HA/M1) VLPs individually displaying H1, H3, $\mathrm{H} 5$, and $\mathrm{H} 7 \mathrm{HA}$ epitopes significantly protected adult mice against an i.n. homologous (H1N1 or H7N1 virus), heterovariant (H5N1 or $\mathrm{H} 7 \mathrm{~N} 9$ virus), or heterosubtypic (H2N1, H7N1, H10N1, or H11N1 virus) challenge (142). The VLP cocktail protected mice against a challenge with the H7N9 or H10N1 virus until 6 months post-vaccination. Passive serum transfer experiments suggested an involvement of anti-HA Abs in the resistance against homologous or heterovariant challenge; however the correlates of protection against the heterosubtypic challenge remain unknown (142). Interestingly, a partial protection was also observed in aged mice against the H10N1 challenge (142).

Other VLP platforms presenting conserved IAV epitopes (M2e or epitopes located in HA2) have been successfully tested in pre-clinical tests with the hepatitis B virus core protein $(73,143$, $144)$, the coat protein of bacteriophage $\mathrm{Q} \beta(\mathrm{Q} \beta$-VLPs) (145), the protrusion domain of the capsid protein of norovirus (P-VLPs) $(138,146)$ or the NP of respiratory syncytial virus $\left(\mathrm{N}_{\mathrm{RSV}}\right.$-VLPs) (147) as VLP scaffold.

Experimental studies in mice have allowed a better understanding of the adjuvant mechanisms of $\mathrm{Q} \beta$-VLPs at the mucosal surfaces $(148,149)$. During the self-assembly process, $\mathrm{Q} \beta$-VLPs are packaged with RNA derived from E. coli which typically mediate TLR3/7 signals. A first study concluded that $\mathrm{Q} \beta$-VLPs applied via the i.n. route were captured by alveolar macrophages and lung DCs, transported to the DLN and subsequent mucosal IgA responses required macrophage/DC activation via TLR7 stimulation (148). A second study established that $\mathrm{Q} \beta$-VLPs administered intranasally could also be taken up by the lung $B$ cells, transported to the spleen and delivered into B cell follicles (149). In accordance with these results, mice intranasally immunized with $\mathrm{Q} \beta$-VLPs incorporating M2e epitopes developed significant mucosal and serum M2e-specific humoral responses, and all animals survived a subsequent i.n. lethal H1N1 challenge (145). Besides Q $\beta$-VLPs, P-VLPs are also efficient mucosal delivery platforms for M2e epitopes in mice (138) and in chickens (146). Chickens intranasally immunized with P-VLPs carrying multiple repetitions of an avian M2e consensus sequence showed a significant reduction in virus shedding from the trachea and the cloaca after an i.n. challenge with avian H5N2 or H7N2 viruses (146). However, no anti-M2e 
$\mathrm{Ab}$ were detected in the serum or in the nasal washes and the protective immune mechanisms remain unknown. Finally, in our research group, we have demonstrated that mice intranasally immunized with three repetitions of M2e sequence from a H1N1 virus exposed at the surface of $\mathrm{N}_{\mathrm{RSV}}$-VLPs developed strong local and systemic M2e-specific Ab responses and were successfully protected against an i.n. homologous challenge (147). Only the i.n. route generated mucosal IgA titers and led to the protection of animals (147).

Although most of the aforementioned influenza VLPs trigger potent host immune reactions by themselves, several studies have indicated that the incorporation of immunostimulating molecules such as enterotoxins $(64,73)$, PRR ligands $(78,79$, $81,82,150)$ or cytokines/chemokines (151), either admixed or anchored at the surface of VLPs, enhances the magnitude, the duration and/or the breadth of the protective immune responses.

Clinical trials have attested the safety and the efficacy of influenza VLPs in parenteral vaccination (136). The promising results obtained in mucosal vaccination during experimental animal studies support the rationale for developing influenza VLPs for human mucosal vaccination as well.

\section{Organic Polymers Chitosan}

Chitosan are natural cationic polysaccharides composed of glucosamine and $\mathrm{N}$-acetylglucosamine resulting from the partial deacetylation of chitin (152). Diverse forms of chitosan are available with various degrees of acetylation, molecular weight and chemical modifications affecting their solubility, biocompatibility, biodegradation and immunogenicity (152). Chitosan polymers and their derivatives have been widely used as mucosal adjuvants under different formulations such as solutions, powders, micro/nanoparticles and gels (152). Because of electrostatic interactions of positively charged chitosan with the negatively charged components of the mucus, chitosan polymers exhibit strong mucoadhesive properties. In addition, chitosan-based adjuvants enhance the permeability of the epithelial barrier by inducing a reversible opening of tight junctions, and consequently promote the interactions of the coadministered $\mathrm{Ag}(\mathrm{s})$ with the MALT. Finally, chitosan polymers directly stimulate the activation and the maturation of APCs including DCs via TLR4-, NLR family pyrin domain containing 3 (NLRP3)-, and/or stimulator of interferon genes (STING)dependent pathway $(152,153)$.

Chitosan-based adjuvants have successfully improved the immunogenicity and the protective potential of i.n. influenza split vaccines in pre-clinical tests (154-157). For example, $N$-trimethyl-derivatives of chitosan (TMC) boosted the immunogenicity of H1N1 split vaccine reflected by increased serum IgG and HAI Ab titers and mucosal IgA titers (154). An optimal immune response was obtained when influenza Ags were conjugated on TMC nanoparticles instead of being encapsulated into the TMC nanoparticles (154). In another study, a thermo-sensitive chitosan-based hydrogel (a solution of $\mathrm{N}$-[(2-hydroxy-3-trimethylammonium) propyl] chitosan chloride (HTCC) plus $\alpha, \beta$-glycerophosphate) formulated with $\mathrm{H} 5 \mathrm{~N} 1$ split vaccine extended the retention time of influenza
Ags in the nasal cavity of mice (156). This could be due to the mucoadhesive properties of HTCC and/or the reduction of the ciliary beating frequency caused by the overlaying of epithelial surface with the semi-solid hydrogel solution (156). In addition, the adjuvant efficiently increased the intercellular penetration of influenza Ags through the mucosa (156). Consequently, mice receiving the hydrogel-adjuvanted vaccine presented a boost in influenza-specific serum and mucosal $\mathrm{Ab}$ titers as well as in cell-mediated immune responses, including an increase in type $1 /$ type 2 cellular responses in the spleen and in the frequency of memory $\mathrm{CD}^{+} \mathrm{T}$ cells in the NALT (156). TMC admixed with $\mathrm{H} 5 \mathrm{~N} 1$ split vaccine also boosted serum Ab titers reactive against the vaccine strain and $\mathrm{H} 5 \mathrm{~N} 1$ heterovariant viruses in ferrets and the adjuvanted vaccine protected animals against a subsequent intratracheal homologous challenge (157).

Pre-clinical experiments in mice and chickens have also revealed that chitosan polymers are efficient mucosal adjuvants for DNA (158) or protein influenza subunit vaccines either admixed with influenza Ags $(144,159)$ or as coating agents on the surface of polyamines $(69,160)$ or polyesters (161, 162) nanoparticles containing influenza Ags (see sections Chitosan and Polyamine polymers). Chitosan-containing lipid nanoparticles complexed with mRNA encoding H5 HA or NP proteins also boosted the immunogenicity of IAV Ags after subcutaneous immunization in mice and rabbits (163). These experiments have to be performed in the context of a mucosal vaccination.

Clinical studies have proven the safety and the adjuvant potential of chitosan for various i.n. vaccines including IIV vaccines, but further investigations are needed $(164,165)$.

\section{Polyamine Polymers}

Poly $(\gamma$-glutamic acid) $(\gamma$-PGA) polymers are anionic biodegradable bacterial polymers with mucoadhesive properties due to the hydrogen bonding between the carboxylate group of $\gamma$-PGA and the hydroxyl group of the mucus glycoproteins (166). In addition, $\gamma$-PGA nanoparticles are efficiently taken up by APCs and they enhance the activation, the maturation and the functions of APCs (167).

Experiments in mice have demonstrated that $\gamma$-PGA polymers are potent mucosal adjuvants/carriers for split or subunit influenza vaccines $(69,160,167,168)$. For example, a first study established that the supplementation of an i.n. H1N1 split vaccine with $\gamma$-PGA nanoparticles enhanced serum and mucosal neutralizing $\mathrm{Ab}$ responses and type 1/type 2-cell mediated immune responses in the spleen (168). These responses were specifically directed against the vaccine strain or a heterovariant virus (168). Mice immunized with the adjuvanted vaccine were fully resistant to i.n. homologous or heterovariant challenge (168). In a second study, the incorporation of

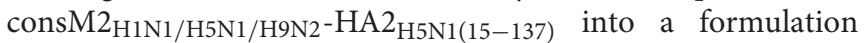
consisting of $\gamma$-PGA polymers associated with MPLA and QS21 (a specific fraction of QuilA saponins) generated higher and more persistent HA2/M2-specific immune responses than a free

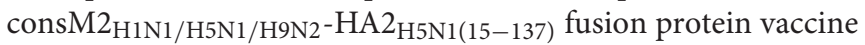
(167). The adjuvanted vaccine conferred a full and/or longlasting protection to mice against an i.n. challenge with different 
subtypes of IAV including H5N1, H1N1, H5N2, or H9N2 viruses (167). Notably, the adjuvant effect of the $\gamma$-PGA/MPLA/QS21 formulation was similar as CT (167).

Polyethyleneimine (PEI) polymers are a family of synthetic cationic molecules widely used as gene transfer agents because of their ability to complex, condense and protect nucleic acids against enzymatic degradation, to promote transfection of cells and to facilitate endosomal escape of nucleic acids (166). In vivo bioluminescence imaging of luciferase performed in mice after the i.n. administration of luciferase-encoding DNA formulated with PEI or unformulated DNA revealed that PEI significantly improved the efficiency of gene transfer in the RT (169). Accordingly, PEI efficiently boosted the magnitude, the breadth and the protective potential of influenza DNA vaccines administered via the i.n. route in mice (169). In particular, the combination of PEI with a DNA vaccine encoding a HA derived from a $\mathrm{H} 5 \mathrm{~N} 1$ virus increased the serum and mucosal $\mathrm{Ab}$ responses against the vaccine strain (169). Furthermore, the animals presented higher frequency of HA-specific memory IFN$\gamma$-secreting $\mathrm{CD}^{+}$and $\mathrm{CD} 8^{+} \mathrm{T}$ cells in the lungs and spleen than mice vaccinated with the naked DNA (169). In accordance with these observations, mice immunized with the adjuvanted vaccine were more resistant to an i.n. homologous challenge (169). Finally, the PEI-complexed vaccine generated cross-reactive immune responses and conferred partial cross-protection to mice against an i.n. H5N1 heterovariant challenge (169). A recent study has demonstrated that PEI conjugated with $\beta$-cyclodextrin (a cyclic polysaccharide) is an excellent platform material for i.n. mRNA vaccination in mice (170). In vivo imaging showed that the complexation of anionic mRNA encoding the envelope glycoprotein gp120 of HIV with the PEI-cyclodextrin polymer prolonged the nasal residence time of the mRNA and increased uptake of the mRNA by nasal epithelial cells (170). In addition, in vitro observations suggested that the polymer facilitated the i.n. delivery of the mRNA cargo through a paracellular route by reversibly opening tight junctions while preserving the integrity of the epithelial barrier (170). Consequently, this polymeric delivery system boosted the gp120-specific serum and mucosal humoral and cellular responses (170). In mice, PEI is a potent adjuvant for subcutaneous or intramuscular mRNA vaccines encoding IAV HA or NP Ags $(171,172)$. However, the ability of PEI to transport IAV mRNA vaccines through the mucosal route awaits further investigation.

Likewise, PEI polymers are potent mucosal adjuvants for influenza WIV and protein subunit vaccines in mice and chickens, with similar potency as bacterial-derived adjuvants (84, 173). In vitro analysis showed that the combination of PEI with H9N2 WIV vaccine increased the adhesion of the viral Ags to Calu-3 epithelial cells (173). In addition PEI enhanced the cellular uptake and endosomal escape of the viral Ags in murine bone marrow-derived DCs, and stimulated the maturation of the cells (173). Accordingly, mice intranasally immunized with the PEIcomplexed WIV vaccine developed higher local and systemic influenza-specific immune responses than mice immunized with the uncomplexed WIV vaccine (173). Finally, a recent study demonstrated $(172,173)$ that chickens immunized with a portion of the HA1 domain of a H7N9 virus formulated with PEI developed higher serum, nasal and lung anti-HA Ab titers than animals immunized with the unadjuvanted formulation (84). The birds also showed reduced viral loads in the cloaca and throat after an i.n. homologous challenge in comparison with the birds of the unadjuvanted group (84).

Because of safety issues related to the non-biodegradable nature of PEI and to the positive charge of these polymers (which can interact electrostatically with cellular anionic macromolecules and interfere with normal cellular functions), less toxic PEI derivatives have been developed such as deacylated PEI. These modified PEI polymers retain adjuvant properties toward HA DNA vaccines in mice (174).

\section{Polyesters}

PLGA, a copolymer of poly (D, L-lactide-co-glycolide) approved for clinical use, has been widely exploited as a nanoparticle delivery system because of its biocompatibility, biodegradability, safety, and controlled release properties (166). Hydrophilic polymeric materials can be added on the surface of PLGA nanoparticles to increase the stability and the transfer of the antigenic payload across mucosal surfaces. These stabilizers are usually polyethylene glycol, polyvinyl alcohol or chitosan (175). Experimental studies in animals have demonstrated that PLGA nanoparticles are good mucosal adjuvants for i.n. IAV WIV vaccines $(162,176)$. A first study showed that chickens immunized with an aerosol vaccine composed of H9N2 WIV admixed with polyvinyl alcohol-modified PLGA nanoparticles incorporating PEI-CpG-ODN complexes generated higher virusspecific serum IgY and HAI Ab titers and mucosal IgA titers than birds immunized with the non-adjuvanted WIV vaccine (176). A second study concluded that chickens immunized via i.n. and intraocular routes with polyvinyl alcohol/chitosan-modified PLGA nanoparticles encapsulating a H4N6 WIV with PEICpG-ODN complexes developed higher influenza-specific serum and mucosal $\mathrm{Ab}$ responses than chickens of the unadjuvanted group (162).

Poly-( $\varepsilon$-caprolactone), another biodegradable and biocompatible polyester polymer, was also described as a potent carrier system for mucosal vaccines against IAV in pre-clinical tests (161). Notably, mice intranasally vaccinated with chitosan-coated poly-( $\varepsilon$-caprolactone) nanoparticles incorporating a recombinant $\mathrm{HA}$ derived from a $\mathrm{H} 1 \mathrm{~N} 1$ virus exhibited higher HA-specific serum and mucosal Ab titers and systemic type 1/type 2-cell-mediated immune responses than mice vaccinated with uncoated nanoparticles or with the unadjuvanted recombinant HA (161).

\section{Other Organic Polymers}

Poly ( $N$-vinylacetamide-co-acrylic acid) (PNVA-co-AA) bearing $\mathrm{D}$-octaarginine are biocompatible cationic oligopeptides which enhance permeation of co-mixed $\mathrm{Ag}(\mathrm{s})$ through mucosal barriers (177). The co-administered $\mathrm{Ag}(\mathrm{s})$ are suggested to be taken up into cells via macropinocytosis through the biorecognition of the peptidyl branches in the polymer backbone, while the polymer remains on the cell membrane (177). The i.n. co-administration of H1N1 WIV vaccine with D-octaarginine-linked PNVA-co-AA 
in mice boosted the HA-specific nasal IgA titers which crossreacted in vitro with recombinant HAs from $\mathrm{H} 1 \mathrm{~N} 1$ heterovariant and H3N2 or H5N1 heterosubtypic viruses $(178,179)$. Mice vaccinated with the adjuvanted preparation showed a better resistance to an i.n. homologous challenge than mice immunized with H1N1 WIV alone $(178,179)$. The cross-protective potential of such polymers remains to be evaluated in vivo.

\section{Inorganic Nanoparticles}

During the last years, gold nanoparticles are appearing increasingly attractive (mucosal) vaccine delivery vehicles (180). As immunologically inert molecules, they do not induce competing carrier-specific immune responses. Also, they can be chemically synthesized with tight control over the nanoparticle size and are easily functionalized with vaccine epitopes/immunopotentiators (180). A series of recent publications has demonstrated that gold nanoparticles are potent mucosal delivery platforms for influenza Ags in mice (81, 82, 181, 182). By designing dual-linker nanoparticles, a research group grafted a recombinant trimeric HA derived from a $\mathrm{H} 3 \mathrm{~N} 2$ virus and a recombinant flagellin monomer on gold nanoparticles by click chemistry and metal-chelating reactions with high conjugation efficiency $(81,82)$. The conjugation of the vaccine epitopes on the nanoparticles increased the uptake of influenza Ags by DCs and stimulated the activation, maturation and function of DCs in vitro (82). With respect to these observations, the vaccine formulation intranasally administered to mice generated stronger influenza-specific immune responses than a vaccine composed of free trimeric HA mixed with flagellin (81). Notably, mice immunized with the nanoparticles showed elevated serum IgG and HAI Ab titers, higher IgG and IgA titers in the URT and LRT and higher frequency of long-lived IgG/IgA ASCs in the spleen (81). In addition, animals exhibited higher influenza-specific systemic and local cell-mediated immune responses. In particular, mice showed a boost in type 1/type 2/type 17-cell-mediated immune responses and in the frequency of CD8 ${ }^{+}$CTLs in the spleen, an increase in type 1-biased cellular responses in the NALT and higher frequency of IFN- $\gamma$-expressing $\mathrm{CD} 4^{+} / \mathrm{CD}^{+} \mathrm{T}$ cells in the DLN (81). All mice survived a lethal i.n. homologous challenge and showed reduced lung viral loads in contrast to animals receiving the vaccine formulation uncoupled to the nanoparticles (81). The attachment of a human M2e consensus sequence to gold nanoparticles also significantly strengthened the immunogenicity and the protective potential of the M2e epitopes in mice (181, 182). Hence, these nanocarriers are interesting mucosal delivery platforms for broadly-protective vaccines against IAV.

\section{Nucleotide-Based Adjuvants}

Polyinosine-polycytidylic acid [poly (I:C)] is a synthetic analog for double-stranded RNA that mimics viral RNA and activates various APCs in a TLR3/Toll or interleukin (IL)-1 receptor domain-containing adaptor-inducing interferon- $\beta$ (TRIF)dependent pathway (61). Numerous studies have demonstrated that the incorporation of poly (I:C) into IAV WIV, split or subunit vaccines enhanced the magnitude and/or the breadth of the specific immune in mice and chickens (183-186).
A recent study showed that the i.n. administration of poly (I:C) with $\mathrm{H} 1 \mathrm{~N} 1$ split vaccine in mice boosted serum $\operatorname{IgG}$ and mucosal IgA Ab responses in a TLR3/TRIF-dependent pathway (183). The adjuvant also increased the frequency of virus-specific IFN- $\gamma$-secreting $\mathrm{CD}^{+}$and $\mathrm{CD}^{+} \mathrm{T}$ cells in the spleen (183). The mucosal adjuvanticity of poly (I:C) relied on its ability to stimulate the activation and the maturation of $\mathrm{CD}_{103}{ }^{+}$ DCs located in the NALT, which were crucially involved in the generation of local humoral and $\mathrm{T}$ cell-mediated immune responses (183). In particular, the addition of poly (I:C) to the vaccine formulation enhanced the frequency of GC B cells and follicular helper T cells in the NALT and the local expression of various cytokines involved in IgA class switching (183). Mice immunized with the adjuvanted vaccine were fully protected against an i.n. H1N1 heterovariant challenge in contrast to $\mathrm{TRIF}^{-/-}$mice or mice immunized with the unadjuvanted vaccine (183).

Poly (I:C) was also tested in a HA2-directed vaccine strategy in mice (185). The methodology consisted of sequential immunizations with different chimeric HA molecules which have the same H1 HA2 but different non-H1 HA1s. The adjuvant boosted the induction of HA2-specific serum IgG and nasal IgA directed against the HA vaccine strain (185). In addition the adjuvant enhanced the generation of serum Abs which cross-reacted with HA molecules from H1N1 heterovariant and H5N1 and H2N2 heterosubtypic viruses (185). The adjuvanted vaccine fully protected mice against i.n. homologous and H1N1 heterovariant challenges (185).

We can mention that poly (I:C) is also an interesting mucosal adjuvant for influenza vaccines in chickens (186). The i.n. coadministration of poly (I:C) with H5N1 IIV vaccine enhanced influenza-specific serum IgG and HAI Ab titers and IgA titers in the nose and in the trachea of birds (186).

Finally, poly (I: $\left.\mathrm{C}_{12} \mathrm{U}\right)$, an analog of poly (I:C) exhibiting a safer profile, gave promising results in pre-clinical (187) and clinical trials (188). In particular, the i.n. immunization of healthy humans with a seasonal LAIV vaccine in association with poly $\left(\mathrm{I}: \mathrm{C}_{12} \mathrm{U}\right.$ ) induced the generation of nasal IgA which reacted with homologous viruses as well as H5N1, H7N9, and H7N3 HPAIV with pandemic potential for humans (188).

Another class of nucleotide-based adjuvant, CDN, exhibited comparable efficacy to poly (I:C) in enhancing the immunogenicity of mucosal IAV split or subunit vaccines in mice (114, 189-192). CDN are bacterial second-messenger molecules detected by the innate immune system via various sensors including STING (189). The inclusion of $2^{\prime}, 3^{\prime}$-cyclic-guanosine monophosphate-adenosine monophosphate (cGAMP) in i.n. H1N1 split vaccine stimulated the activation of innate and adaptive immunity in the NALT, the spleen and the DLN, and promoted GC formation in the NALT in a STINGdependent pathway (189). It resulted in an increase in specific serum and mucosal humoral responses and in systemic type 1/type 2/type 17 cellular immune responses, including higher frequency of IFN- $\gamma$-producing $\mathrm{CD} 4^{+}$and $\mathrm{CD} 8^{+} \mathrm{T}$ cells in the spleen (189). Other studies demonstrated that the addition of $\mathrm{CDN}$ to recombinant $\mathrm{H} 5 \mathrm{~N} 1 \mathrm{HA}$ or H1N1 NP subunit vaccines enhanced the magnitude and/or the breadth of the 
humoral and cellular anti-IAV immune responses in mice $(190,192)$.

CPG-ODN are TLR9/TLR21 agonists which activate various APCs and commonly generate pro-inflammatory and Th1biaised immune milieu (193). These compounds have thus been widely exploited as vaccine enhancers in numerous preclinical and human clinical trials (193). Several studies in mice and chickens have demonstrated that CpG-ODN alone or in combination with other immunopotentiators/delivery systems are suitable mucosal adjuvants for IAV WIV, split or subunit vaccines $(64,83,99,176,181,182,186,194-$ 197). By using an in vitro DCs:Calu-3 cells co-culture model and by doing i.n. instillation in mice in vivo, a recent study proposed a mechanism for the mucosal adjuvanticity of CpGODN toward a H9N2 WIV vaccine. The authors suggested that CpG-ODN stimulated the secretion of the chemokine (C-C motif) ligand 20 (CCL20) by epithelial cells in a TLR9-dependent pathway, which enhanced DC recruitment to the nasal epithelium and promoted the formation of transepithelial dendrites involved in the capture of luminal influenza Ags (197). Clinical trials have proven the safety and immunogenicity of CpG-ODN-adjuvanted IIV vaccines when administered via the parenteral route (85). In view of the very promising results in animal models, the efficacy of CpGODN in mucosal anti-IAV vaccination should be evaluated in humans.

\section{Cytokines/Chemokines}

Cytokines and chemokines are signaling molecules released by a wide range of immune and nonimmune cells (198). They are critically involved in host defense against infections by modulating the activation, maturation, differentiation, migration, survival and functions of innate and adaptive immune cells (198). These molecules have been considered as interesting alternatives to pathogen-derived adjuvants for anti-IAV vaccines (198).

Various cytokines such as IL-2, IL-12, IL-1 family cytokines (IL- $1 \alpha / \beta$, IL-18, and IL-33), IL-23 and type I IFNs are efficient and safe mucosal adjuvants when admixed with IAV WIV, split or subunit vaccines in mice $(198,199)$. For example, the incorporation of type I IFNs to an i.n. H1N1 split vaccine enhanced the magnitude and the duration of virus-specific serum and mucosal humoral responses and increased the protection of mice against an i.n. homologous challenge (200). The mucosal adjuvanticity of type I IFNs was suggested to be related to the ability of the cytokines to stimulate the uptake of co-administered Ags by phagocytes of the nasal mucosa (200). In another study in mice, the i.n. co-administration of cytokines belonging to the IL-1 family with recombinant HA derived from a H1N1 virus increased the magnitude of serum and mucosal anti-HA Abs titers in serum and in mucosal secretions as well as the frequency of type 1/type 2 cytokine-producing cells and CD8 ${ }^{+}$CTLs in the spleen (201). The adjuvant remarkably enhanced the level of protection of mice against an i.n. heterovariant challenge (201). Mucosal vaccine formulations with cytokines/chemokines anchored into influenza WIV or VLPs have also been successfully tested in mice (151, 202-204). The incorporation of cytokines/chemokines in nanoparticle vaccine formulations could overcome the relatively short half-life of these molecules (198). Recent studies have been focused on CCL28, which is highly expressed by epithelial cells and selectively attracts $\mathrm{T}$ and $\mathrm{B}$ cell subsets (including IgA ASCs) at mucosal surfaces $(151,203)$. CCL28 anchored in H3N2 (HA/M1) VLPs showed in vitro chemotactic activity toward chemokine (C-C motif) receptor 3 (CCR3)/CCR10-expressing mouse splenocytes and lung cells (203). In mice, the chemokine enhanced the magnitude, the avidity, the functionality and the duration of serum and mucosal humoral responses specifically directed against homologous and heterovariant viruses $(151,203)$. Accordingly, the adjuvanted vaccine conferred a higher and more persistent protection in animals against an i.n. homologous or heterovariant challenge (151, 203).

Although cytokines/chemokines are promising adjuvants for mucosal anti-IAV vaccines, these molecules may present some drawbacks (198). The pleiotropic effects of these proteins on immune cells may result in unwanted adverse effects. In addition, the adjuvant effects observed in animal models may not be directly translated to humans as it was reported for an i.n. influenza split vaccine formulated with type I IFNs (205).

\section{Adjuvants and Trained Immunity}

Studies listed so far in this review have evaluated the efficacy of mucosal adjuvants on their ability to enhance adaptive immune responses ( $\mathrm{T}$ and/or B cell responses) specifically directed against influenza Ags (Supplemental Table 1). However, a growing body of evidence suggests that some categories of adjuvants may also induce long-lasting functional state within innate immune cells, resulting in an increase in nonspecific host defense against a range of pathogens. This concept has been named "trained immunity" (206). Some studies have indicated that prophylactic mucosal administration of bacterial-derived molecules, in particular enterotoxins, TLR3 and TLR9 ligands, or type I IFNs protected mice against a subsequent IAV challenge (73, 207-209). For example, mice intranasally treated with CT, LT (R192G) or CpG-ODN showed reduced mortality and/or lung viral loads subsequently to a H1N1 challenge $24 \mathrm{~h}$ after the last treatment. The improved resistance was associated with higher frequencies of $\mathrm{CD}^{+}$ $\mathrm{T}$ cells, B cells and DCs in the airways and the generation of BALT-like structures in the lungs (207). Furthermore, the i.n. administration of chitosan fully protected mice against lethal challenge with H7N9 or diverse H1N1 viruses (210). The protection was associated with infiltration of leukocytes in the bronchoalveolar lavage and an enhanced expression of inflammatory cytokines in the LRT (210). Mice were significantly protected against H7N9 challenge even 10 days after the chitosan administration (210). Whether the protective efficacy of the mucosal IAV vaccine formulations compiled in this review was influenced by trained immunity remains to be determined. 


\section{CONCLUSIONS}

Vaccination via the i.n. route has the potential to elicit longlasting and cross-protective humoral and cellular immune responses at the portal of entry of respiratory pathogens. It is thus a suitable strategy to prevent infections caused by highly variable IAV. However, the i.n. vaccine formulations have to be rationally elaborated because the respiratory mucosal surfaces restrain the immunoavailability and the immunogenicity of vaccine $\mathrm{Ag}(\mathrm{s})$. Numerous experimental animal studies have demonstrated the promising potential of various adjuvant/delivery systems for the development of i.n. human and veterinary (chickens) vaccines against IAV. They consist of immunopotentiatory molecules, such as bacterial-derived components, synthetic nucleotides and cytokines/chemokines, associated or not with particulate carriers, such as lipid-based particles, VLPs, organic polymers and inorganic nanoparticles. These adjuvant systems boost the efficacy of current IIV vaccines or new subunit vaccine candidates by increasing the magnitude, the persistence and/or the breadth of the host (protective) anti-IAV immunity. Some of these novel mucosal vaccine formulations are safe and immunogenic in early phase clinical trials but they have to overcome several hurdles before reaching the market, including regulatory and economic restrictions and the lack of appropriate correlates of protection. Standardized assays taking into account the protective role of non-HAI Abs and cell-mediated immunity need to be developed to adequately evaluate the efficacy of these new mucosal formulations. Needlefree mucosal vaccines that provide a broad-coverage against IAV and do not require annual re-vaccination are promising

\section{REFERENCES}

1. Rajao DS, Perez DR. Universal vaccines and vaccine platforms to protect against influenza viruses in humans and agriculture. Front Microbiol. (2018) 9:123. doi: 10.3389/fmicb.2018.00123

2. Organization WH. Influenza (Seasonal) Fact Sheet. (2017). Available online at: https://www.who.int/news-room/fact-sheets/detail/influenza-(seasonal)

3. Pantin-Jackwood MJ, Swayne DE. Pathogenesis and pathobiology of avian influenza virus infection in birds. Rev Sci Tech. (2009) 28:113-36. doi: 10.20506/rst.28.1.1869

4. Tamura S, Ainai A, Suzuki T, Kurata T, Hasegawa H. Intranasal inactivated influenza vaccines: a reasonable approach to improve the efficacy of influenza vaccine? Jpn J Infect Dis. (2016) 69:165-79. doi: 10.7883/yoken.JJID.2015.560

5. Cerutti A, Chen K, Chorny A. Immunoglobulin responses at the mucosal interface. Annu Rev Immunol. (2011) 29:273-93. doi: 10.1146/annurev-immunol-031210-101317

6. de Geus ED, Rebel JM, Vervelde L. Induction of respiratory immune responses in the chicken; implications for development of mucosal avian influenza virus vaccines. Vet Q. (2012) 32:75-86. doi: 10.1080/01652176.2012.711956

7. Pulendran B, Maddur MS. Innate immune sensing and response to influenza. Curr Top Microbiol Immunol. (2015) 386:23-71. doi: 10.1007/82_2014_405

8. Vangeti S, Yu M, Smed-Sorensen A. Respiratory mononuclear phagocytes in human influenza A virus infection: their role in immune protection and as targets of the virus. Front Immunol. (2018) 9:1521. doi: 10.3389/fimmu.2018.01521

9. Oshansky CM, Thomas PG. The human side of influenza. J Leukoc Biol. (2012) 92:83-96. doi: 10.1189/jlb.1011506 alternatives to current IAV vaccines and should increase awareness about the benefits of influenza vaccination among the general public.

\section{AUTHOR CONTRIBUTIONS}

$\mathrm{CCa}$ and $\mathrm{CCh}$ have written, prepared, and approved the manuscript for publication.

\section{FUNDING}

This work was supported by a grant from the Livestock Vaccine Innovation Fund (LVIF) (Canada's International Development Research Centre, Bill \& Melinda Gates Foundation, Global Affairs Canada).

\section{ACKNOWLEDGMENTS}

This work was supported by a grant from the International Development Research Center (IDRC) to CCh. We thank Dr. Jean Millet (VIM, INRA, Jouy-en-Josas) for critical reading of the manuscript.

\section{SUPPLEMENTARY MATERIAL}

The Supplementary Material for this article can be found online at: https://www.frontiersin.org/articles/10.3389/fimmu. 2019.01605/full\#supplementary-material

Supplemental Table 1 | Adjuvants/delivery systems tested in intranasal vaccination against IAV infections in pre-clinical and clinical studies.

10. GeurtsvanKessel CH, Willart MA, van Rijt LS, Muskens F, Kool M, Baas $\mathrm{C}$, et al. Clearance of influenza virus from the lung depends on migratory langerin ${ }^{+} \mathrm{CD}_{11 b^{-}}$but not plasmacytoid dendritic cells. J Exp Med. (2008) 205:1621-34. doi: 10.1084/jem.20071365

11. Helft J, Manicassamy B, Guermonprez P, Hashimoto D, Silvin A, Agudo J, et al. Cross-presenting $\mathrm{CD}_{103^{+}}$dendritic cells are protected from influenza virus infection. J Clin Invest. (2012) 122:4037-47. doi: 10.1172/JCI60659

12. Ho AW, Prabhu N, Betts RJ, Ge MQ, Dai X, Hutchinson PE, et al. Lung $\mathrm{CD} 103^{+}$dendritic cells efficiently transport influenza virus to the lymph node and load viral antigen onto MHC class I for presentation to CD8 T cells. J Immunol. (2011) 187:6011-21. doi: 10.4049/jimmunol.1100987

13. Kim TS, Braciale TJ. Respiratory dendritic cell subsets differ in their capacity to support the induction of virus-specific cytotoxic $\mathrm{CD} 8^{+} \mathrm{T}$ cell responses. PLoS ONE. (2009) 4:e4204. doi: 10.1371/journal.pone.0004204

14. Ballesteros-Tato A, Leon B, Lund FE, Randall TD. Temporal changes in dendritic cell subsets, cross-priming and costimulation via CD70 control $\mathrm{CD}^{+} \mathrm{T}$ cell responses to influenza. Nat Immunol. (2010) 11:216-24. doi: $10.1038 /$ ni. 1838

15. Belz GT, Smith CM, Kleinert L, Reading P, Brooks A, Shortman K, et al. Distinct migrating and nonmigrating dendritic cell populations are involved in MHC class I-restricted antigen presentation after lung infection with virus. Proc Natl Acad Sci USA. (2004) 101:8670-5. doi: 10.1073/pnas.0402644101

16. Aldridge JR Jr., Moseley CE, Boltz DA, Negovetich NJ, Reynolds C, Franks J, et al. TNF/iNOS-producing dendritic cells are the necessary evil of lethal influenza virus infection. Proc Natl Acad Sci USA. (2009) 106:5306-11. doi: 10.1073/pnas.0900655106

17. Jego G, Palucka AK, Blanck JP, Chalouni C, Pascual V, Banchereau J. Plasmacytoid dendritic cells induce plasma cell differentiation through 
type I interferon and interleukin 6. Immunity. (2003) 19:225-34. doi: 10.1016/S1074-7613(03)00208-5

18. Hemann EA, Sjaastad LE, Langlois RA, Legge KL. Plasmacytoid dendritic cells require direct infection to sustain the pulmonary influenza A virus-specific CD8 T Cell Response. J Virol. (2015) 90:2830-7. doi: 10.1128/JVI.02546-15

19. McGill J, Van Rooijen N, Legge KL. Protective influenza-specific CD8 T cell responses require interactions with dendritic cells in the lungs. J Exp Med. (2008) 205:1635-46. doi: 10.1084/jem.20080314

20. Cole SL, Ho LP. Contribution of innate immune cells to pathogenesis of severe influenza virus infection. Clin Sci. (2017) 131:269-83. doi: 10.1042/CS20160484

21. Lawrence CW, Braciale TJ. Activation, differentiation, and migration of naive virus-specific $\mathrm{CD}^{+} \mathrm{T}$ cells during pulmonary influenza virus infection. $J$ Immunol. (2004) 173:1209-18. doi: 10.4049/jimmunol.173.2.1209

22. Roman E, Miller E, Harmsen A, Wiley J, Von Andrian UH, Huston $\mathrm{G}$, et al. CD4 effector $\mathrm{T}$ cell subsets in the response to influenza: heterogeneity, migration, and function. J Exp Med. (2002) 196:957-68. doi: $10.1084 /$ jem.20021052

23. Topham DJ, Tripp RA, Doherty PC. $\mathrm{CD}^{+} \mathrm{T}$ cells clear influenza virus by perforin or Fas-dependent processes. J Immunol. (1997) 159:5197-200.

24. Brincks EL, Katewa A, Kucaba TA, Griffith TS, Legge KL. CD8 T cells utilize TRAIL to control influenza virus infection. J Immunol. (2008) 181:4918-25. doi: 10.4049/jimmunol.181.7.4918

25. Hua L, Yao S, Pham D, Jiang L, Wright J, Sawant D, et al. Cytokine-dependent induction of $\mathrm{CD}^{+} \mathrm{T}$ cells with cytotoxic potential during influenza virus infection. J Virol. (2013) 87:11884-93. doi: 10.1128/JVI.01461-13

26. Brown DM, Lee S, Garcia-Hernandez Mde L, Swain SL. Multifunctional CD4 cells expressing gamma interferon and perforin mediate protection against lethal influenza virus infection. J Virol. (2012) 86:6792-803. doi: 10.1128/JVI.07172-11

27. Brown DM, Dilzer AM, Meents DL, Swain SL. CD4 T cell-mediated protection from lethal influenza: perforin and antibody-mediated mechanisms give a one-two punch. J Immunol. (2006) 177:2888-98. doi: 10.4049/jimmunol.177.5.2888

28. Wilkinson TM, Li CK, Chui CS, Huang AK, Perkins M, Liebner JC, et al. Preexisting influenza-specific $\mathrm{CD} 4^{+} \mathrm{T}$ cells correlate with disease protection against influenza challenge in humans. Nat Med. (2012) 18:27480. doi: $10.1038 / \mathrm{nm} .2612$

29. Sridhar S, Begom S, Bermingham A, Hoschler K, Adamson W, Carman W, et al. Cellular immune correlates of protection against symptomatic pandemic influenza. Nat Med. (2013) 19:1305-12. doi: 10.1038/nm.3350

30. McMichael AJ, Gotch FM, Noble GR, Beare PA. Cytotoxic Tcell immunity to influenza. $N$ Engl J Med. (1983) 309:13-7. doi: 10.1056/NEJM198307073090103

31. Kumar A, Meldgaard TS, Bertholet S. Novel platforms for the development of a universal influenza vaccine. Front Immunol. (2018) 9:600. doi: 10.3389/fimmu.2018.00600

32. Sant AJ, DiPiazza AT, Nayak JL, Rattan A, Richards KA. CD4 T cells in protection from influenza virus: viral antigen specificity and functional potential. Immunol Rev. (2018) 284:91-105. doi: 10.1111/imr.12662

33. Sant AJ, Richards KA, Nayak J. Distinct and complementary roles of CD4 T cells in protective immunity to influenza virus. Curr Opin Immunol. (2018) 53:13-21. doi: 10.1016/j.coi.2018.03.019

34. Takahashi Y, Onodera T, Adachi Y, Ato M. Adaptive B cell responses to influenza virus infection in the lung. Viral Immunol. (2017) 30:431-7. doi: 10.1089/vim.2017.0025

35. Boyden AW, Legge KL, Waldschmidt TJ. Pulmonary infection with influenza A virus induces site-specific germinal center and $\mathrm{T}$ follicular helper cell responses. PLoS ONE. (2012) 7:e40733. doi: 10.1371/journal.pone.0040733

36. Joo HM, He Y, Sangster MY. Broad dispersion and lung localization of virusspecific memory B cells induced by influenza pneumonia. Proc Natl Acad Sci USA. (2008) 105:3485-90. doi: 10.1073/pnas.0800003105

37. Hasegawa H, van Reit E, Kida H. Mucosal immunization and adjuvants. Curr Top Microbiol Immunol. (2015) 386:371-80. doi: 10.1007/82_2014_402

38. He W, Mullarkey CE, Duty JA, Moran TM, Palese P, Miller MS. Broadly neutralizing anti-influenza virus antibodies: enhancement of neutralizing potency in polyclonal mixtures and IgA backbones. J Virol. (2015) 89:3610-8. doi: 10.1128/JVI.03099-14

39. Renegar KB, Small PA Jr., Boykins LG, Wright PF. Role of IgA versus IgG in the control of influenza viral infection in the murine respiratory tract. $J$ Immunol. (2004) 173:1978-86. doi: 10.4049/jimmunol.173.3.1978

40. Gould VMW, Francis JN, Anderson KJ, Georges B, Cope AV, Tregoning JS. Nasal IgA provides protection against human influenza challenge in volunteers with low serum influenza antibody titre. Front Microbiol. (2017) 8:900. doi: $10.3389 /$ fmicb. 2017.00900

41. Krammer F. The human antibody response to influenza A virus infection and vaccination. Nat Rev Immunol. (2019). 19:383-97 doi: 10.1038/s41577-019-0143-6

42. Corti D, Cameroni E, Guarino B, Kallewaard NL, Zhu Q, Lanzavecchia A. Tackling influenza with broadly neutralizing antibodies. Curr Opin Virol. (2017) 24:60-9. doi: 10.1016/j.coviro.2017.03.002

43. Jegaskanda S. The potential role of Fc-receptor functions in the development of a universal influenza vaccine. Vaccines. (2018) 6:27 doi: $10.3390 /$ vaccines 6020027

44. Jegaskanda S, Vanderven HA, Wheatley AK, Kent SJ. Fc or not Fc; that is the question: antibody Fc-receptor interactions are key to universal influenza vaccine design. Hum Vaccin Immunother. (2017) 13:1-9. doi: 10.1080/21645515.2017.1290018

45. Vanderven HA, Jegaskanda S, Wheatley AK, Kent SJ. Antibody-dependent cellular cytotoxicity and influenza virus. Curr Opin Virol. (2017) 22:89-96. doi: 10.1016/j.coviro.2016.12.002

46. Wu T, Hu Y, Lee YT, Bouchard KR, Benechet A, Khanna K, et al. Lungresident memory $\mathrm{CD} 8 \mathrm{~T}$ cells. $\left(\mathrm{T}_{\mathrm{RM}}\right)$ are indispensable for optimal crossprotection against pulmonary virus infection. J Leukoc Biol. (2014) 95:21524. doi: $10.1189 / \mathrm{jlb} .0313180$

47. Slutter B, Van Braeckel-Budimir N, Abboud G, Varga SM, Salek-Ardakani S, Harty JT. Dynamics of influenza-induced lung-resident memory T cells underlie waning heterosubtypic immunity. Sci Immunol. (2017) 2:eaag2031 doi: 10.1126/sciimmunol.aag2031

48. Pizzolla A, Nguyen THO, Smith JM, Brooks AG, Kedzieska K, Heath WR, et al. Resident memory $\mathrm{CD} 8^{+} \mathrm{T}$ cells in the upper respiratory tract prevent pulmonary influenza virus infection. Sci Immunol. (2017) 2:eaam6970 doi: 10.1126/sciimmunol.aam6970

49. Turner DL, Bickham KL, Thome JJ, Kim CY, D'Ovidio F, Wherry EJ, et al. Lung niches for the generation and maintenance of tissue-resident memory T cells. Mucosal Immunol. (2014) 7:501-10. doi: 10.1038/mi.2013.67

50. Teijaro JR, Turner D, Pham Q, Wherry EJ, Lefrancois L, Farber DL. Cutting edge: tissue-retentive lung memory $\mathrm{CD} 4 \mathrm{~T}$ cells mediate optimal protection to respiratory virus infection. J Immunol. (2011) 187:5510-4. doi: 10.4049/jimmunol.1102243

51. Sridhar S. Heterosubtypic T-Cell immunity to influenza in humans: challenges for universal T-cell influenza vaccines. Front Immunol. (2016) 7:195. doi: $10.3389 /$ fimmu.2016.00195

52. Adachi $\mathrm{Y}$, Onodera T, Yamada $\mathrm{Y}$, Daio R, Tsuiji M, Inoue T, et al. Distinct germinal center selection at local sites shapes memory B cell response to viral escape. J Exp Med. (2015) 212:1709-23. doi: 10.1084/jem.20142284

53. Zens KD, Chen JK, Farber DL. Vaccine-generated lung tissue-resident memory $\mathrm{T}$ cells provide heterosubtypic protection to influenza infection. JCI Insight. (2016) 1:e85832 doi: 10.1172/jci.insight.85832

54. Suarez DL, Pantin-Jackwood MJ. Recombinant viral-vectored vaccines for the control of avian influenza in poultry. Vet Microbiol. (2017) 206:144-51. doi: 10.1016/j.vetmic.2016.11.025

55. de Vries RD, Rimmelzwaan GF. Viral vector-based influenza vaccines. Hum Vaccin Immunother. (2016) 12:2881-901. doi: 10.1080/21645515.2016.1210729

56. Lee LYY, Izzard L, Hurt AC. A review of DNA vaccines against influenza. Front Immunol. (2018) 9:1568. doi: 10.3389/fimmu.2018.01568

57. Pardi N, Hogan MJ, Porter FW, Weissman D. mRNA vaccinesa new era in vaccinology. Nat Rev Drug Discov. (2018) 17:261-79. doi: 10.1038/nrd.2017.243

58. Zhang C, Maruggi G, Shan H, Li J. Advances in mRNA vaccines for infectious diseases. Front Immunol. (2019) 10:594. doi: 10.3389/fimmu.2019.00594

59. Awate S, Babiuk LA, Mutwiri G. Mechanisms of action of adjuvants. Front Immunol. (2013) 4:114. doi: 10.3389/fimmu.2013.00114 
60. Aoshi T. Modes of action for mucosal vaccine adjuvants. Viral Immunol. (2017) 30:463-70. doi: 10.1089/vim.2017.0026

61. Apostolico Jde S, Lunardelli VA, Coirada FC, Boscardin SB, Rosa DS. Adjuvants: classification, modus operandi, and licensing. I Immunol Res. (2016) 2016:1459394. doi: 10.1155/2016/1459394

62. Woodrow KA, Bennett KM, Lo DD. Mucosal vaccine design and delivery. Annu Rev Biomed Eng. (2012) 14:17-46. doi: 10.1146/annurev-bioeng-071811-150054

63. Lycke N, Lebrero-Fernandez C. ADP-ribosylating enterotoxins as vaccine adjuvants. Curr Opin Pharmacol. (2018) 41:42-51. doi: 10.1016/j.coph.2018.03.015

64. Quan FS, Ko EJ, Kwon YM, Joo KH, Compans RW, Kang SM. Mucosal adjuvants for influenza virus-like particle vaccine. Viral Immunol. (2013) 26:385-95. doi: 10.1089/vim.2013.0013

65. Quan FS, Compans RW, Nguyen HH, Kang SM. Induction of heterosubtypic immunity to influenza virus by intranasal immunization. J Virol. (2008) 82:1350-9. doi: 10.1128/JVI.01615-07

66. Tumpey TM, Renshaw M, Clements JD, Katz JM. Mucosal delivery of inactivated influenza vaccine induces B-cell-dependent heterosubtypic crossprotection against lethal influenza A H5N1 virus infection. J Virol. (2001) 75:5141-50. doi: 10.1128/JVI.75.11.5141-5150.2001

67. Quan FS, Yoo DG, Song JM, Clements JD, Compans RW, Kang SM. Kinetics of immune responses to influenza virus-like particles and dosedependence of protection with a single vaccination. J Virol. (2009) 83:448997. doi: 10.1128/JVI.02035-08

68. Zhang Y, Yu X, Hou L, Chen J, Li P, Qiao X, et al. CTA1: Purified and display onto gram-positive enhancer matrix (GEM) particles as mucosal adjuvant. Protein Expr Purif. (2018) 141:19-24. doi: 10.1016/j.pep.2017.08.010

69. Chowdhury MYE, Kim TH, Uddin MB, Kim JH, Hewawaduge CY, Ferdowshi Z, et al. Mucosal vaccination of conserved sM2, HA2 and cholera toxin subunit A1 (CTA1) fusion protein with poly gammaglutamate/chitosan nanoparticles (PC NPs) induces protection against divergent influenza subtypes. Vet Microbiol. (2017) 201:240-51. doi: 10.1016/j.vetmic.2017.01.020

70. Helgeby A, Robson NC, Donachie AM, Beackock-Sharp H, Lovgren $\mathrm{K}$, Schon $\mathrm{K}$, et al. The combined CTA1-DD/ISCOM adjuvant vector promotes priming of mucosal and systemic immunity to incorporated antigens by specific targeting of B cells. J Immunol. (2006) 176:3697-706. doi: 10.4049/jimmunol.176.6.3697

71. Eliasson DG, El Bakkouri K, Schon K, Ramne A, Festjens E, Lowenadler B, et al. CTA1-M2e-DD: a novel mucosal adjuvant targeted influenza vaccine. Vaccine. (2008) 26:1243-52. doi: 10.1016/j.vaccine.2007.12.027

72. Bernasconi V, Bernocchi B, Ye L, Le MQ, Omokanye A, Carpentier $\mathrm{R}$, et al. Porous nanoparticles with self-adjuvanting M2e-fusion protein and recombinant hemagglutinin provide strong and broadly protective immunity against influenza virus infections. Front Immunol. (2018) 9:2060. doi: 10.3389/fimmu.2018.02060

73. De Filette M, Ramne A, Birkett A, Lycke N, Lowenadler B, Min Jou W, et al. The universal influenza vaccine $\mathrm{M} 2 \mathrm{e}-\mathrm{HBc}$ administered intranasally in combination with the adjuvant CTA1-DD provides complete protection. Vaccine. (2006) 24:544-51. doi: 10.1016/j.vaccine.2005.08.061

74. Hajam IA, Dar PA, Shahnawaz I, Jaume JC, Lee JH. Bacterial flagellina potent immunomodulatory agent. Exp Mol Med. (2017) 49:e373. doi: $10.1038 / \mathrm{emm} .2017 .172$

75. Chabot SM, Shawi M, Eaves-Pyles T, Neutra MR. Effects of flagellin on the functions of follicle-associated epithelium. J Infect Dis. (2008) 198:907-10. doi: $10.1086 / 591056$

76. Skountzou I, Martin Mdel P, Wang B, Ye L, Koutsonanos D, Weldon $\mathrm{W}$, et al. Salmonella flagellins are potent adjuvants for intranasally administered whole inactivated influenza vaccine. Vaccine. (2010) 28:410312. doi: 10.1016/j.vaccine.2009.07.058

77. Hong SH, Byun YH, Nguyen CT, Kim SY, Seong BL, Park S, et al. Intranasal administration of a flagellin-adjuvanted inactivated influenza vaccine enhances mucosal immune responses to protect mice against lethal infection. Vaccine. (2012) 30:466-74. doi: 10.1016/j.vaccine.2011. 10.058

78. Wang BZ, Xu R, Quan FS, Kang SM, Wang L, Compans RW. Intranasal immunization with influenza VLPs incorporating membrane-anchored flagellin induces strong heterosubtypic protection. PLoS ONE. (2010) 5:e13972. doi: 10.1371/journal.pone.0013972

79. Wang L, Wang YC, Feng H, Ahmed T, Compans RW, Wang BZ. Virus-like particles containing the tetrameric ectodomain of influenza matrix protein 2 and flagellin induce heterosubtypic protection in mice. Biomed Res Int. (2013) 2013:686549. doi: 10.1155/2013/686549

80. Wang BZ, Gill HS, He C, Ou C, Wang L, Wang YC, et al. Microneedle delivery of an M2e-TLR5 ligand fusion protein to skin confers broadly cross-protective influenza immunity. J Control Release. (2014) 178:1-7. doi: 10.1016/j.jconrel.2014.01.002

81. Wang C, Zhu W, Luo Y, Wang BZ. Gold nanoparticles conjugating recombinant influenza hemagglutinin trimers and flagellin enhanced mucosal cellular immunity. Nanomedicine. (2018) 14:1349-60. doi: 10.1016/j.nano.2018.03.007

82. Wang C, Zhu W, Wang BZ. Dual-linker gold nanoparticles as adjuvanting carriers for multivalent display of recombinant influenza hemagglutinin trimers and flagellin improve the immunological responses in vivo and in vitro. Int J Nanomedicine. (2017) 12:4747-62. doi: 10.2147/IJN.S137222

83. Chaung HC, Cheng LT, Hung LH, Tsai PC, Skountzou I, Wang B, et al. Salmonella flagellin enhances mucosal immunity of avian influenza vaccine in chickens. Vet Microbiol. (2012) 157:69-77. doi: 10.1016/j.vetmic.2011.12.014

84. Song L, Xiong D, Song H, Wu L, Zhang M, Kang X, et al. Mucosal and systemic immune responses to influenza H7N9 antigen HA1-2 co-delivered intranasally with flagellin or polyethyleneimine in mice and chickens. Front Immunol. (2017) 8:326. doi: 10.3389/fimmu.2017.00326

85. Tregoning JS, Russell RF, Kinnear E. Adjuvanted influenza vaccines. Hum Vaccin Immunother. (2018) 14:550-64. doi: 10.1080/21645515.2017.1415684

86. Turley CB, Rupp RE, Johnson C, Taylor DN, Wolfson J, Tussey L, et al. Safety and immunogenicity of a recombinant M2e-flagellin influenza vaccine (STF2.4xM2e) in healthy adults. Vaccine. (2011) 29:5145-52. doi: 10.1016/j.vaccine.2011.05.041

87. Burt D, Mallett C, Plante M, Zimmermann J, Torossian K, Fries L. Proteosome-adjuvanted intranasal influenza vaccines: advantages, progress and future considerations. Expert Rev Vaccines. (2011) 10:365-75. doi: $10.1586 /$ erv.10.172

88. Plante M, Jones T, Allard F, Torossian K, Gauthier J, St-Felix N, et al. Nasal immunization with subunit proteosome influenza vaccines induces serum HAI, mucosal IgA and protection against influenza challenge. Vaccine. (2001) 20:218-25. doi: 10.1016/S0264-410X(01)00268-7

89. Jones T, Cyr S, Allard F, Bellerose N, Lowell GH, Burt DS. Protollin: a novel adjuvant for intranasal vaccines. Vaccine. (2004) 22:3691-7. doi: 10.1016/j.vaccine.2004.03.035

90. Jones T, Allard F, Cyr SL, Tran SP, Plante M, Gauthier J, et al. A nasal Proteosome influenza vaccine containing baculovirus-derived hemagglutinin induces protective mucosal and systemic immunity. Vaccine. (2003) 21:3706-12. doi: 10.1016/S0264-410X(03)00387-6

91. Lambkin-Williams R, Gelder C, Broughton R, Mallett CP, Gilbert AS, Mann $A$, et al. An intranasal proteosome-adjuvanted trivalent influenza vaccine is safe, immunogenic \& efficacious in the human viral influenza challenge model. Serum IgG \& mucosal IgA are important correlates of protection against illness associated with infection. PLoS ONE. (2016) 11:e0163089. doi: 10.1371/journal.pone.0163089

92. Van Braeckel-Budimir N, Haijema BJ, Leenhouts K. Bacterium-like particles for efficient immune stimulation of existing vaccines and new subunit vaccines in mucosal applications. Front Immunol. (2013) 4:282. doi: 10.3389/fimmu.2013.00282

93. Saluja V, Amorij JP, van Roosmalen ML, Leenhouts K, Huckriede A, Hinrichs WL, et al. Intranasal delivery of influenza subunit vaccine formulated with GEM particles as an adjuvant. AAPS J. (2010) 12:109-16. doi: 10.1208/s12248-009-9168-2

94. de Haan A, Haijema BJ, Voorn P, Meijerhof T, van Roosmalen ML, Leenhouts K. Bacterium-like particles supplemented with inactivated influenza antigen induce cross-protective influenza-specific antibody responses through intranasal administration. Vaccine. (2012) 30:4884-91. doi: 10.1016/j.vaccine.2012.04.032

95. Keijzer C, Haijema BJ, Meijerhof T, Voorn P, de Haan A, Leenhouts K, et al. Inactivated influenza vaccine adjuvanted with bacterium-like particles 
induce systemic and mucosal influenza A virus specific T-cell and B-cell responses after nasal administration in a TLR2 dependent fashion. Vaccine. (2014) 32:2904-10. doi: 10.1016/j.vaccine.2014.02.019

96. Lee TY, Kim CU, Bae EH, Seo SH, Jeong DG, Yoon SW, et al. Outer membrane vesicles harboring modified lipid A moiety augment the efficacy of an influenza vaccine exhibiting reduced endotoxicity in a mouse model. Vaccine. (2017) 35:586-95. doi: 10.1016/j.vaccine.2016.12.025

97. Bernasconi V, Norling K, Bally M, Hook F, Lycke NY. Mucosal vaccine development based on liposome technology. J Immunol Res. (2016) 2016:5482087. doi: 10.1155/2016/5482087

98. Corthesy B, Bioley G. Lipid-Based Particles: versatile delivery systems for mucosal vaccination against infection. Front Immunol. (2018) 9:431. doi: 10.3389/fimmu.2018.00431

99. Joseph A, Louria-Hayon I, Plis-Finarov A, Zeira E, Zakay-Rones Z, Raz E, et al. Liposomal immunostimulatory DNA sequence (ISS-ODN): an efficient parenteral and mucosal adjuvant for influenza and hepatitis B vaccines. Vaccine. (2002) 20:3342-54. doi: 10.1016/S0264-410X(02)00295-5

100. Qu W, Li N, Yu R, Zuo W, Fu T, Fei W, et al. Cationic DDA/TDB liposome as a mucosal vaccine adjuvant for uptake by dendritic cells in vitro induces potent humoural immunity. Artif Cells Nanomed Biotechnol. 46:852-60. doi: 10.1080/21691401.2018.1438450

101. Christensen D, Foged C, Rosenkrands I, Lundberg CV, Andersen P, Agger EM, et al. CAF01 liposomes as a mucosal vaccine adjuvant: in vitro and in vivo investigations. Int J Pharm. (2010) 390:19-24. doi: 10.1016/j.ijpharm.2009.10.043

102. Even-Or O, Joseph A, Itskovitz-Cooper N, Samira S, Rochlin E, Eliyahu $\mathrm{H}$, et al. A new intranasal influenza vaccine based on a novel polycationic lipid-ceramide carbamoyl-spermine (CCS). II. Studies in mice and ferrets and mechanism of adjuvanticity. Vaccine. (2011) 29:2474-86. doi: 10.1016/j.vaccine.2011.01.009

103. Joseph A, Itskovitz-Cooper N, Samira S, Flasterstein O, Eliyahu $\mathrm{H}$, Simberg $\mathrm{D}$, et al. A new intranasal influenza vaccine based on a novel polycationic lipid-ceramide carbamoyl-spermine (CCS) I. Immunogenicity and efficacy studies in mice. Vaccine. (2006) 24:3990-4006. doi: 10.1016/j.vaccine.2005.12.017

104. Even-Or O, Samira S, Ellis R, Kedar E, Barenholz Y. Adjuvanted influenza vaccines. Expert Rev Vaccines. (2013) 12:1095-108. doi: 10.1586/14760584.2013.825445

105. Wang D, Christopher ME, Nagata LP, Zabielski MA, Li H, Wong JP, et al. Intranasal immunization with liposome-encapsulated plasmid DNA encoding influenza virus hemagglutinin elicits mucosal, cellular and humoral immune responses. J Clin Virol. (2004) 31(Suppl. 1):S99-106. doi: 10.1016/j.jcv.2004.09.013

106. Ninomiya A, Ogasawara K, Kajino K, Takada A, Kida H. Intranasal administration of a synthetic peptide vaccine encapsulated in liposome together with an anti-CD40 antibody induces protective immunity against influenza A virus in mice. Vaccine. (2002) 20:3123-9. doi: 10.1016/S0264-410X(02)00261-X

107. Adler-Moore J, Munoz M, Kim H, Romero J, Tumpey T, Zeng H, et al. Characterization of the murine Th2 response to immunization with liposomal M2e influenza vaccine. Vaccine. (2011) 29:4460-8. doi: 10.1016/j.vaccine.2011.04.040

108. Scorza FB, Pardi N. New kids on the block: RNA-based influenza virus vaccines. Vaccines. (2018) 6:20 doi: 10.3390/vaccines6020020

109. Reichmuth AM, Oberli MA, Jaklenec A, Langer R, Blankschtein D. mRNA vaccine delivery using lipid nanoparticles. Ther Deliv. (2016) 7:319-34. doi: 10.4155/tde-2016-0006

110. Magini D, Giovani C, Mangiavacchi S, Maccari S, Cecchi R, Ulmer $\mathrm{JB}$, et al. Self-amplifying mRNA vaccines expressing multiple conserved influenza antigens confer protection against homologous and heterosubtypic viral challenge. PLoS ONE. (2016) 11:e0161193. doi: 10.1371/journal.pone.0161193

111. Bahl K, Senn JJ, Yuzhakov O, Bulychev A, Brito LA, Hassett KJ, et al. Preclinical and clinical demonstration of immunogenicity by mRNA vaccines against H10N8 and H7N9 influenza viruses. Mol Ther. (2017) 25:1316-27. doi: 10.1016/j.ymthe.2017.03.035

112. Pardi N, Parkhouse K, Kirkpatrick E, McMahon M, Zost SJ, Mui BL, et al. Nucleoside-modified mRNA immunization elicits influenza virus hemagglutinin stalk-specific antibodies. Nat Commun. (2018) 9:3361. doi: 10.1038/s41467-018-05482-0

113. Phua KK, Staats HF, Leong KW, Nair SK. Intranasal mRNA nanoparticle vaccination induces prophylactic and therapeutic anti-tumor immunity. Sci Rep. (2014) 4:5128. doi: 10.1038/srep05128

114. Ebensen T, Debarry J, Pedersen GK, Blazejewska P, Weissmann S, Schulze K, et al. Mucosal administration of cycle-di-nucleotide-adjuvanted virosomes efficiently induces protection against influenza $\mathrm{H} 5 \mathrm{~N} 1$ in mice. Front Immunol. (2017) 8:1223. doi: 10.3389/fimmu.2017.01223

115. Moser C, Muller M, Kaeser MD, Weydemann U, Amacker M. Influenza virosomes as vaccine adjuvant and carrier system. Expert Rev Vaccines. (2013) 12:779-91. doi: 10.1586/14760584.2013.811195

116. Drane D, Gittleson C, Boyle J, Maraskovsky E. ISCOMATRIX adjuvant for prophylactic and therapeutic vaccines. Expert Rev Vaccines. (2007) 6:761-72. doi: 10.1586/14760584.6.5.761

117. Eliasson DG, Helgeby A, Schon K, Nygren C, El-Bakkouri K, Fiers W, et al. A novel non-toxic combined CTA1-DD and ISCOMS adjuvant vector for effective mucosal immunization against influenza virus. Vaccine. (2011) 29:3951-61. doi: 10.1016/j.vaccine.2011.03.090

118. Coulter A, Harris R, Davis R, Drane D, Cox J, Ryan D, et al. Intranasal vaccination with ISCOMATRIX adjuvanted influenza vaccine. Vaccine. (2003) 21:946-9. doi: 10.1016/S0264-410X(02)00545-5

119. Suartha IN, Suartini GAA, Wirata IW, Dewi N, Putra GNN, Kencana GAY, et al. Intranasal administration of inactivated avian influenza virus of H5N1 subtype vaccine-induced systemic immune response in chicken and mice. Vet World. (2018) 11:221-6. doi: 10.14202/vetworld.2018.221-226

120. Mizuno D, Ide-Kurihara M, Ichinomiya T, Kubo I, Kido H. Modified pulmonary surfactant is a potent adjuvant that stimulates the mucosal IgA production in response to the influenza virus antigen. J Immunol. (2006) 176:1122-30. doi: 10.4049/jimmunol.176.2.1122

121. Mizuno D, Kimoto T, Takei T, Fukuta A, Shinahara W, Takahashi E, et al. Surfactant protein $\mathrm{C}$ is an essential constituent for mucosal adjuvanticity of Surfacten, acting as an antigen delivery vehicle and inducing both local and systemic immunity. Vaccine. (2011) 29:5368-78. doi: 10.1016/j.vaccine.2011.05.090

122. Kimoto T, Mizuno D, Takei T, Kunimi T, Ono S, Sakai S, et al. Intranasal influenza vaccination using a new synthetic mucosal adjuvant SF-10: induction of potent local and systemic immunity with balanced Th1 and Th2 responses. Influenza Other Respir Viruses. (2013) 7:1218-26. doi: 10.1111/irv.12124

123. Kim H, Kimoto $T$, Sakai S, Takahashi E, Kido H. Adjuvanting influenza hemagglutinin vaccine with a human pulmonary surfactantmimicking synthetic compound SF-10 induces local and systemic cell-mediated immunity in mice. PLoS ONE. (2018) 13:e0191133. doi: 10.1371/journal.pone.0191133

124. Mizuno D, Kimoto T, Sakai S, Takahashi E, Kim H, Kido H. Induction of systemic and mucosal immunity and maintenance of its memory against influenza A virus by nasal vaccination using a new mucosal adjuvant SF-10 derived from pulmonary surfactant in young cynomolgus monkeys. Vaccine. (2016) 34:1881-8. doi: 10.1016/j.vaccine.2016.02.061

125. Maltais AK, Stittelaar KJ, Veldhuis Kroeze EJ, van Amerongen G, Dijkshoorn ML, Krestin GP, et al. Intranasally administered Endocine formulated 2009 pandemic influenza H1N1 vaccine induces broad specific antibody responses and confers protection in ferrets. Vaccine. (2014) 32:3307-15. doi: 10.1016/j.vaccine.2014.03.061

126. Falkeborn T, Brave A, Larsson M, Akerlind B, Schroder U, Hinkula J. Endocine, N3OA and N3OASq; three mucosal adjuvants that enhance the immune response to nasal influenza vaccination. PLoS ONE. (2013) 8:e70527. doi: 10.1371/journal.pone.00 70527

127. Falkeborn T, Hinkula J, Olliver M, Lindberg A, Maltais AK. The intranasal adjuvant Endocine enhances both systemic and mucosal immune responses in aged mice immunized with influenza antigen. Virol J. (2017) 14:44. doi: 10.1186/s12985-017-0698-4

128. Makidon PE, Belyakov IM, Blanco LP, Janczak KW, Landers J, Bielinska $\mathrm{AU}$, et al. Nanoemulsion mucosal adjuvant uniquely activates cytokine production by nasal ciliated epithelium and induces dendritic cell trafficking. Eur J Immunol. (2012) 42:2073-86. doi: 10.1002/eji.201142346 
129. Myc A, Kukowska-Latallo JF, Smith DM, Passmore C, Pham T, Wong $\mathrm{P}$, et al. Nanoemulsion nasal adjuvant $\mathrm{W}_{80} 5 \mathrm{EC}$ induces dendritic cell engulfment of antigen-primed epithelial cells. Vaccine. (2013) 31:1072-9. doi: 10.1016/j.vaccine.2012.12.033

130. Bielinska AU, Gerber M, Blanco LP, Makidon PE, Janczak KW, Beer $\mathrm{M}$, et al. Induction of Th17 cellular immunity with a novel nanoemulsion adjuvant. Crit Rev Immunol. (2010) 30:189-99. doi: 10.1615/CritRevImmunol.v30.i2.60

131. Bielinska AU, Makidon PE, Janczak KW, Blanco LP, Swanson B, Smith DM, et al. Distinct pathways of humoral and cellular immunity induced with the mucosal administration of a nanoemulsion adjuvant. J Immunol. (2014) 192:2722-33. doi: 10.4049/jimmunol.1301424

132. Das SC, Hatta M, Wilker PR, Myc A, Hamouda T, Neumann G, et al. Nanoemulsion $\mathrm{W}_{80} 5 \mathrm{EC}$ improves immune responses upon intranasal delivery of an inactivated pandemic $\mathrm{H} 1 \mathrm{~N} 1$ influenza vaccine. Vaccine. (2012) 30:6871-7. doi: 10.1016/j.vaccine.2012.09.007

133. Hamouda T, Sutcliffe JA, Ciotti S, Baker JR Jr. Intranasal immunization of ferrets with commercial trivalent influenza vaccines formulated in a nanoemulsion-based adjuvant. Clin Vaccine Immunol. (2011) 18:1167-75. doi: 10.1128/CVI.00035-11

134. Stanberry LR, Simon JK, Johnson C, Robinson PL, Morry J, Flack MR, et al. Safety and immunogenicity of a novel nanoemulsion mucosal adjuvant $\mathrm{W}_{80} 5 \mathrm{EC}$ combined with approved seasonal influenza antigens. Vaccine. (2012) 30:307-16. doi: 10.1016/j.vaccine.2011.10.094

135. Brazzoli M, Magini D, Bonci A, Buccato S, Giovani C, Kratzer R, et al. Induction of broad-based immunity and protective efficacy by selfamplifying mRNA vaccines encoding influenza virus hemagglutinin. J Virol. (2016) 90:332-44. doi: 10.1128/JVI.01786-15

136. Quan FS, Lee YT, Kim KH, Kim MC, Kang SM. Progress in developing viruslike particle influenza vaccines. Expert Rev Vaccines. (2016) 15:1281-93. doi: 10.1080/14760584.2016.1175942

137. Kang SM, Song JM, Quan FS, Compans RW. Influenza vaccines based on virus-like particles. Virus Res. (2009) 143:140-6. doi: 10.1016/j.virusres.2009.04.005

138. Xia M, Tan M, Wei C, Zhong W, Wang L, McNeal M, et al. A candidate dual vaccine against influenza and noroviruses. Vaccine. (2011) 29:7670-7. doi: 10.1016/j.vaccine.2011.07.139

139. Perrone LA, Ahmad A, Veguilla V, Lu X, Smith G, Katz JM, et al. Intranasal vaccination with 1918 influenza virus-like particles protects mice and ferrets from lethal 1918 and H5N1 influenza virus challenge. J Virol. (2009) 83:572634. doi: 10.1128/JVI.00207-09

140. Quan FS, Huang C, Compans RW, Kang SM. Virus-like particle vaccine induces protective immunity against homologous and heterologous strains of influenza virus. J Virol. (2007) 81:3514-24. doi: 10.1128/JVI.02052-06

141. Song JM, Wang BZ, Park KM, Van Rooijen N, Quan FS, Kim MC, et al. Influenza virus-like particles containing M2 induce broadly cross protective immunity. PLoS ONE. (2011) 6:e14538. doi: 10.1371/journal.pone.0014538

142. Schwartzman LM, Cathcart AL, Pujanauski LM, Qi L, Kash JC, Taubenberger JK. An intranasal virus-like particle vaccine broadly protects mice from multiple subtypes of influenza A virus. MBio. (2015) 6:e01044. doi: $10.1128 / \mathrm{mBio} .01044-15$

143. Chen S, Zheng D, Li C, Zhang W, Xu W, Liu X, et al. Protection against multiple subtypes of influenza viruses by virus-like particle vaccines based on a hemagglutinin conserved epitope. Biomed Res Int. (2015) 2015:901817. doi: 10.1155/2015/901817

144. Zheng D, Chen S, Qu D, Chen J, Wang F, Zhang R, et al. Influenza H7N9 $\mathrm{LAH}-\mathrm{HBc}$ virus-like particle vaccine with adjuvant protects mice against homologous and heterologous influenza viruses. Vaccine. (2016) 34:6464-71. doi: 10.1016/j.vaccine.2016.11.026

145. Bessa J, Schmitz N, Hinton HJ, Schwarz K, Jegerlehner A, Bachmann MF. Efficient induction of mucosal and systemic immune responses by viruslike particles administered intranasally: implications for vaccine design. Eur J Immunol. (2008) 38:114-26. doi: 10.1002/eji.200636959

146. Elaish M, Kang KI, Xia M, Ali A, Shany SA, Wang L, et al. Immunogenicity and protective efficacy of the norovirus $\mathrm{P}$ particle-M2e chimeric vaccine in chickens. Vaccine. (2015) 33:4901-9. doi: 10.1016/j.vaccine.2015.07.049

147. Herve PL, Raliou M, Bourdieu C, Dubuquoy C, Petit-Camurdan A, Bertho $\mathrm{N}$, et al. A novel subnucleocapsid nanoplatform for mucosal vaccination against influenza virus that targets the ectodomain of matrix protein $2 . J$ Virol. (2014) 88:325-38. doi: 10.1128/JVI.01141-13

148. Bessa J, Jegerlehner A, Hinton HJ, Pumpens P, Saudan P, Schneider $\mathrm{P}$, et al. Alveolar macrophages and lung dendritic cells sense RNA and drive mucosal IgA responses. J Immunol. (2009) 183:3788-99. doi: 10.4049/jimmunol.0804004

149. Bessa J, Zabel F, Link A, Jegerlehner A, Hinton HJ, Schmitz N, et al. Lowaffinity B cells transport viral particles from the lung to the spleen to initiate antibody responses. Proc Natl Acad Sci USA. (2012) 109:20566-71. doi: 10.1073/pnas.1206970109

150. Schneider-Ohrum K, Giles BM, Weirback HK, Williams BL, DeAlmeida DR, Ross TM. Adjuvants that stimulate TLR3 or NLPR3 pathways enhance the efficiency of influenza virus-like particle vaccines in aged mice. Vaccine. (2011) 29:9081-92. doi: 10.1016/j.vaccine.2011.09.051

151. Mohan T, Deng L, Wang BZ. CCL28 chemokine: an anchoring point bridging innate and adaptive immunity. Int Immunopharmacol. (2017) 51:165-70. doi: 10.1016/j.intimp.2017.08.012

152. Xia Y, Fan Q, Hao D, Wu J, Ma G, Su Z. Chitosan-based mucosal adjuvants: sunrise on the ocean. Vaccine. (2015) 33:5997-6010. doi: 10.1016/j.vaccine.2015.07.101

153. Carroll EC, Jin L, Mori A, Munoz-Wolf N, Oleszycka E, Moran HBT, et al. The vaccine adjuvant chitosan promotes cellular immunity via DNA sensor cGAS-STING-dependent induction of type I interferons. Immunity. (2016) 44:597-608. doi: 10.1016/j.immuni.2016.02.004

154. Liu Q, Zheng X, Zhang C, Shao X, Zhang X, Zhang Q, et al. Conjugating influenza A. (H1N1) antigen to $\mathrm{N}$-trimethylaminoethylmethacrylate chitosan nanoparticles improves the immunogenicity of the antigen after nasal administration. J Med Virol. (2015) 87:1807-15. doi: 10.1002/jmv.24253

155. Amidi M, Romeijn SG, Verhoef JC, Junginger HE, Bungener L, Huckriede A, et al. N-trimethyl chitosan (TMC) nanoparticles loaded with influenza subunit antigen for intranasal vaccination: biological properties and immunogenicity in a mouse model. Vaccine. (2007) 25:144-53. doi: 10.1016/j.vaccine.2006.06.086

156. Wu Y, Wei W, Zhou M, Wang Y, Wu J, Ma G, et al. Thermalsensitive hydrogel as adjuvant-free vaccine delivery system for H5N1 intranasal immunization. Biomaterials. (2012) 33:2351-60. doi: 10.1016/j.biomaterials.2011.11.068

157. Mann AJ, Noulin N, Catchpole A, Stittelaar KJ, de Waal L, Veldhuis Kroeze EJ, et al. Intranasal $\mathrm{H} 5 \mathrm{~N} 1$ vaccines, adjuvanted with chitosan derivatives, protect ferrets against highly pathogenic influenza intranasal and intratracheal challenge. PLoS ONE. (2014) 9:e93761. doi: 10.1371/journal.pone.0093761

158. Sawaengsak C, Mori Y, Yamanishi K, Srimanote P, Chaicumpa W, Mitrevej $A$, et al. Intranasal chitosan-DNA vaccines that protect across influenza virus subtypes. Int J Pharm. (2014) 473:113-25. doi: 10.1016/j.ijpharm.2014. 07.005

159. Sui Z, Chen Q, Wu R, Zhang H, Zheng M, Wang H, et al. Crossprotection against influenza virus infection by intranasal administration of M2-based vaccine with chitosan as an adjuvant. Arch Virol. (2010) 155:53544. doi: 10.1007/s00705-010-0621-4

160. Moon HJ, Lee JS, Talactac MR, Chowdhury MY, Kim JH, Park ME, et al. Mucosal immunization with recombinant influenza hemagglutinin protein and poly gamma-glutamate/chitosan nanoparticles induces protection against highly pathogenic influenza A virus. Vet Microbiol. (2012) 160:27789. doi: 10.1016/j.vetmic.2012.05.035

161. Gupta NK, Tomar P, Sharma V, Dixit VK. Development and characterization of chitosan coated poly-( $\varepsilon$-caprolactone) nanoparticulate system for effective immunization against influenza. Vaccine. (2011) 29:9026-37. doi: 10.1016/j.vaccine.2011.09.033

162. Alkie TN, Yitbarek A, Taha-Abdelaziz K, Astill J, Sharif S. Characterization of immunogenicity of avian influenza antigens encapsulated in PLGA nanoparticles following mucosal and subcutaneous delivery in chickens. PLoS ONE. (2018) 13:e0206324. doi: 10.1371/journal.pone.0206324

163. McCullough KC, Bassi I, Milona P, Suter R, Thomann-Harwood L, Englezou $\mathrm{P}$, et al. Self-replicating replicon-RNA delivery to dendritic cells by chitosannanoparticles for translation in vitro and in vivo. Mol Ther Nucleic Acids. (2014) 3:e173. doi: 10.1038/mtna.2014.24 
164. Read RC, Naylor SC, Potter CW, Bond J, Jabbal-Gill I, Fisher A, et al. Effective nasal influenza vaccine delivery using chitosan. Vaccine. (2005) 23:4367-74. doi: 10.1016/j.vaccine.2005.04.021

165. Smith A, Perelman M, Hinchcliffe M. Chitosan: a promising safe and immune-enhancing adjuvant for intranasal vaccines. Hum Vaccin Immunother. (2014) 10:797-807. doi: 10.4161/hv.27449

166. Jia Y, Krishnan L, Omri A. Nasal and pulmonary vaccine delivery using particulate carriers. Expert Opin Drug Deliv. (2015) 12:993-1008. doi: 10.1517/17425247.2015.1044435

167. Noh HJ, Chowdhury MY, Cho S, Kim JH, Park HS, Kim CJ, et al. Programming of influenza vaccine broadness and persistence by mucoadhesive polymer-based adjuvant systems. I Immunol. (2015) 195:2472-82. doi: 10.4049/jimmunol.1500492

168. Okamoto S, Matsuura M, Akagi T, Akashi M, Tanimoto T, Ishikawa T, et al. Poly $(\gamma$-glutamic acid) nano-particles combined with mucosal influenza virus hemagglutinin vaccine protects against influenza virus infection in mice. Vaccine. (2009) 27:5896-905. doi: 10.1016/j.vaccine.2009.07.037

169. Torrieri-Dramard L, Lambrecht B, Ferreira HL, Van den Berg T, Klatzmann D, Bellier B. Intranasal DNA vaccination induces potent mucosal and systemic immune responses and cross-protective immunity against influenza viruses. Mol Ther. (2011) 19:602-11. doi: 10.1038/mt.2010.222

170. Li M, Zhao M, Fu Y, Li Y, Gong T, Zhang Z, et al. Enhanced intranasal delivery of mRNA vaccine by overcoming the nasal epithelial barrier via intra- and paracellular pathways. J Control Release. (2016) 228:9-19. doi: 10.1016/j.jconrel.2016.02.043

171. Demoulins T, Milona P, Englezou PC, Ebensen T, Schulze K, Suter R, et al. Polyethylenimine-based polyplex delivery of self-replicating RNA vaccines. Nanomedicine. (2016) 12:711-22. doi: 10.1016/j.nano.2015.11.001

172. Vogel AB, Lambert L, Kinnear E, Busse D, Erbar S, Reuter KC, et al. SelfAmplifying RNA vaccines give equivalent protection against influenza to mRNA vaccines but at much lower doses. Mol Ther. (2018) 26:446-55. doi: 10.1016/j.ymthe.2017.11.017

173. Qin T, Yin Y, Huang L, Yu Q, Yang Q. H9N2 influenza whole inactivated virus combined with polyethyleneimine strongly enhances mucosal and systemic immunity after intranasal immunization in mice. Clin Vaccine Immunol. (2015) 22:421-9. doi: 10.1128/CVI.00778-14

174. Mann JF, McKay PF, Arokiasamy S, Patel RK, Klein K, Shattock RJ. Pulmonary delivery of DNA vaccine constructs using deacylated PEI elicits immune responses and protects against viral challenge infection. J Control Release. (2013) 170:452-9. doi: 10.1016/j.jconrel.2013.06.004

175. Csaba N, Garcia-Fuentes M, Alonso MJ. Nanoparticles for nasal vaccination. Adv Drug Deliv Rev. (2009) 61:140-57. doi: 10.1016/j.addr.2008.09.005

176. Singh SM, Alkie TN, Abdelaziz KT, Hodgins DC, Novy A, Nagy E, et al. Characterization of immune responses to an inactivated avian influenza virus vaccine adjuvanted with nanoparticles containing CpG ODN. Viral Immunol. (2016) 29:269-75. doi: 10.1089/vim.2015.0144

177. Sakuma S, Suita M, Yamamoto T, Masaoka Y, Kataoka M, Yamashita S, et al. Performance of cell-penetrating peptide-linked polymers physically mixed with poorly membrane-permeable molecules on cell membranes. Eur J Pharm Biopharm. (2012) 81:64-73. doi: 10.1016/j.ejpb.2012. 01.008

178. Sakuma S, Suita M, Inoue S, Marui Y, Nishida K, Masaoka Y, et al. Cellpenetrating peptide-linked polymers as carriers for mucosal vaccine delivery. Mol Pharm. (2012) 9:2933-41. doi: 10.1021/mp300329r

179. Miyata K, Mohri K, Egawa T, Endo R, Morimoto N, Ochiai K, et al. Demonstration of D-octaarginine-linked polymers as promising adjuvants for mucosal vaccination through influenza virus challenge. Bioconjug Chem. (2016) 27:1865-71. doi: 10.1021/acs.bioconjchem.6b00283

180. Salazar-Gonzalez JA, Gonzalez-Ortega O, Rosales-Mendoza S. Gold nanoparticles and vaccine development. Expert Rev Vaccines. (2015) 14:1197-211. doi: 10.1586/14760584.2015.1064772

181. Tao W, Ziemer KS, Gill HS. Gold nanoparticle-M2e conjugate coformulated with $\mathrm{CpG}$ induces protective immunity against influenza A virus. Nanomedicine. (2014) 9:237-51. doi: 10.2217/nnm.13.58

182. Tao W, Hurst BL, Shakya AK, Uddin MJ, Ingrole RS, Hernandez-Sanabria $\mathrm{M}$, et al. Consensus M2e peptide conjugated to gold nanoparticles confers protection against $\mathrm{H} 1 \mathrm{~N} 1, \mathrm{H} 3 \mathrm{~N} 2$ and $\mathrm{H} 5 \mathrm{~N} 1$ influenza A viruses. Antiviral Res. (2017) 141:62-72. doi: 10.1016/j.antiviral.2017.01.021
183. Takaki H, Kure S, Oshiumi H, Sakoda Y, Suzuki T, Ainai A, et al. Toll-like receptor 3 in nasal $\mathrm{CD}_{103}{ }^{+}$dendritic cells is involved in immunoglobulin A production. Mucosal Immunol. (2018) 11:82-96. doi: 10.1038/mi.2017.48

184. Ichinohe T, Watanabe I, Ito S, Fujii H, Moriyama M, Tamura S, et al. Synthetic double-stranded RNA poly(I:C) combined with mucosal vaccine protects against influenza virus infection. J Virol. (2005) 79:2910-9. doi: 10.1128/JVI.79.5.2910-2919.2005

185. Goff PH, Eggink D, Seibert CW, Hai R, Martinez-Gil L, Krammer F, et al. Adjuvants and immunization strategies to induce influenza virus hemagglutinin stalk antibodies. PLoS ONE. (2013) 8:e79194. doi: 10.1371/journal.pone.0079194

186. Liang J, Fu J, Kang H, Lin J, Yu Q, Yang Q. Comparison of 3 kinds of Toll-like receptor ligands for inactivated avian H5N1 influenza virus intranasal immunization in chicken. Poult Sci. (2013) 92:2651-60. doi: $10.3382 /$ ps.2013-03193

187. Ichinohe T, Tamura S, Kawaguchi A, Ninomiya A, Imai M, Itamura S, et al. Cross-protection against H5N1 influenza virus infection is afforded by intranasal inoculation with seasonal trivalent inactivated influenza vaccine. $J$ Infect Dis. (2007) 196:1313-20. doi: 10.1086/521304

188. Overton ET, Goepfert PA, Cunningham P, Carter WA, Horvath J, Young $\mathrm{D}$, et al. Intranasal seasonal influenza vaccine and a TLR-3 agonist, rintatolimod, induced cross-reactive IgA antibody formation against avian H5N1 and H7N9 influenza HA in humans. Vaccine. (2014) 32:5490-5. doi: 10.1016/j.vaccine.2014.07.078

189. Takaki H, Takashima K, Oshiumi H, Ainai A, Suzuki T, Hasegawa $\mathrm{H}$, et al. cGAMP promotes germinal center formation and production of IgA in nasal-associated lymphoid tissue. Med Sci. (2017) 5:35 doi: $10.3390 /$ medsci5040035

190. Madhun AS, Haaheim LR, Nostbakken JK, Ebensen T, Chichester J, Yusibov V, et al. Intranasal c-di-GMP-adjuvanted plant-derived H5 influenza vaccine induces multifunctional Th1 $\mathrm{CD}^{+}$cells and strong mucosal and systemic antibody responses in mice. Vaccine. (2011) 29:4973-82. doi: 10.1016/j.vaccine.2011.04.094

191. Svindland SC, Pedersen GK, Pathirana RD, Bredholt G, Nostbakken JK, JulLarsen A, et al. A study of chitosan and c-di-GMP as mucosal adjuvants for intranasal influenza H5N1 vaccine. Influenza Other Respir Viruses. (2013) 7:1181-93. doi: 10.1111/irv.12056

192. Sanchez MV, Ebensen T, Schulze K, Cargnelutti D, Blazejewska P, Scodeller $\mathrm{EA}$, et al. Intranasal delivery of influenza rNP adjuvanted with cdi-AMP induces strong humoral and cellular immune responses and provides protection against virus challenge. PLoS ONE. (2014) 9:e104824. doi: 10.1371/journal.pone.0104824

193. Klinman DM. Immunotherapeutic uses of CpG oligodeoxynucleotides. Nat Rev Immunol. (2004) 4:249-58. doi: 10.1038/nri1329

194. Moldoveanu Z, Love-Homan L, Huang WQ, Krieg AM. CpG DNA, a novel immune enhancer for systemic and mucosal immunization with influenza virus. Vaccine. (1998) 16:1216-24. doi: 10.1016/S0264-410X(98)80122-9

195. Fu J, Liang J, Kang H, Lin J, Yu Q, Yang Q. Effects of different CpG oligodeoxynucleotides with inactivated avian $\mathrm{H} 5 \mathrm{~N} 1$ influenza virus on mucosal immunity of chickens. Poult Sci. (2013) 92:2866-75. doi: 10.3382/ps.2013-03205

196. Zacharias ZR, Ross KA, Hornick EE, Goodman JT, Narasimhan B, Waldschmidt TJ, et al. Polyanhydride nanovaccine induces robust pulmonary $\mathrm{B}$ and $\mathrm{T}$ cell immunity and confers protection against homologous and heterologous influenza A virus infections. Front Immunol. (2018) 9:1953. doi: 10.3389/fimmu.2018.01953

197. Qin T, Yin Y, Yu Q, Huang L, Wang X, Lin J, et al. CpG oligodeoxynucleotides facilitate delivery of whole inactivated H9N2 influenza virus via transepithelial dendrites of dendritic cells in nasal mucosa. $J$ Virol. (2015) 89:5904-18. doi: 10.1128/JVI.00296-15

198. Wang X, Meng D. Innate endogenous adjuvants prime to desirable immune responses via mucosal routes. Protein Cell. (2015) 6:170-84. doi: $10.1007 / \mathrm{s} 13238-014-0125-1$

199. Arulanandam BP, O’Toole M, Metzger DW. Intranasal interleukin-12 is a powerful adjuvant for protective mucosal immunity. J Infect Dis. (1999) 180:940-9. doi: 10.1086/314996

200. Bracci L, Canini I, Puzelli S, Sestili P, Venditti M, Spada M, et al. Type I IFN is a powerful mucosal adjuvant for a selective intranasal vaccination against 
influenza virus in mice and affects antigen capture at mucosal level. Vaccine. (2005) 23:2994-3004. doi: 10.1016/j.vaccine.2004.12.006

201. Kayamuro H, Yoshioka Y, Abe Y, Arita S, Katayama K, Nomura T, et al. Interleukin-1 family cytokines as mucosal vaccine adjuvants for induction of protective immunity against influenza virus. J Virol. (2010) 84:12703-12. doi: 10.1128/JVI.01182-10

202. Khan T, Heffron CL, High KP, Roberts PC. Membrane-bound IL12 and IL-23 serve as potent mucosal adjuvants when co-presented on whole inactivated influenza vaccines. Virol J. (2014) 11:78. doi: 10.1186/1743-422X-11-78

203. Mohan T, Kim J, Berman Z, Wang S, Compans RW, Wang BZ. Co-delivery of GPI-anchored CCL28 and influenza HA in chimeric virus-like particles induces cross-protective immunity against $\mathrm{H} 3 \mathrm{~N} 2$ viruses. J Control Release. (2016) 233:208-19. doi: 10.1016/j.jconrel.2016.05.021

204. Liu J, Ren Z, Wang H, Zhao Y, Wilker PR, Yu Z, et al. Influenza virus-like particles composed of conserved influenza proteins and GPIanchored CCL28/GM-CSF fusion proteins enhance protective immunity against homologous and heterologous viruses. Int Immunopharmacol. (2018) 63:119-28. doi: 10.1016/j.intimp.2018.07.011

205. Couch RB, Atmar RL, Cate TR, Quarles JM, Keitel WA, Arden NH, et al. Contrasting effects of type I interferon as a mucosal adjuvant for influenza vaccine in mice and humans. Vaccine. (2009) 27:5344-8. doi: 10.1016/j.vaccine.2009.06.084

206. Sanchez-Ramon S, Conejero L, Netea MG, Sancho D, Palomares O, Subiza JL. Trained immunity-based vaccines: a new paradigm for the development of broad-spectrum anti-infectious formulations. Front Immunol. (2018) 9:2936. doi: $10.3389 /$ fimmu.2018.02936
207. Norton EB, Clements JD, Voss TG, Cardenas-Freytag L. Prophylactic administration of bacterially derived immunomodulators improves the outcome of influenza virus infection in a murine model. $J$ Virol. (2010) 84:2983-95. doi: 10.1128/JVI.01805-09

208. Beilharz MW, Cummins JM, Bennett AL. Protection from lethal influenza virus challenge by oral type 1 interferon. Biochem Biophys Res Commun. (2007) 355:740-4. doi: 10.1016/j.bbrc.2007. 02.019

209. Lau YF, Tang LH, Ooi EE. A TLR3 ligand that exhibits potent inhibition of influenza virus replication and has strong adjuvant activity has the potential for dual applications in an influenza pandemic. Vaccine. (2009) 27:1354-64. doi: 10.1016/j.vaccine.2008.12.048

210. Zheng $M, Q u ~ D$, Wang $H$, Sun $Z$, Liu $X$, Chen J, et al. Intranasal administration of chitosan against influenza A (H7N9) virus infection in a mouse model. Sci Rep. (2016) 6:28729. doi: 10.1038/srep 28729

Conflict of Interest Statement: The authors declare that the research was conducted in the absence of any commercial or financial relationships that could be construed as a potential conflict of interest.

Copyright $\odot 2019$ Calzas and Chevalier. This is an open-access article distributed under the terms of the Creative Commons Attribution License (CC BY). The use, distribution or reproduction in other forums is permitted, provided the original author(s) and the copyright owner(s) are credited and that the original publication in this journal is cited, in accordance with accepted academic practice. No use, distribution or reproduction is permitted which does not comply with these terms. 